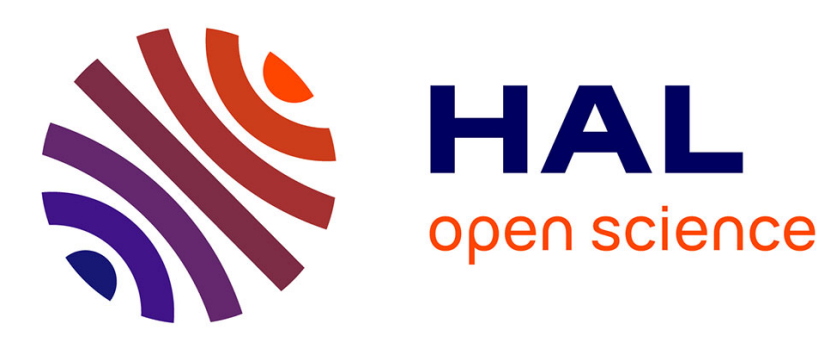

\title{
Joint Design of Emission Tax and Trading Systems
}

Bernard Caillaud, Gabrielle Demange

\section{To cite this version:}

Bernard Caillaud, Gabrielle Demange. Joint Design of Emission Tax and Trading Systems. 2016. halshs-01112185v2

\section{HAL Id: halshs-01112185 \\ https://shs.hal.science/halshs-01112185v2}

Preprint submitted on 1 Jul 2016

HAL is a multi-disciplinary open access archive for the deposit and dissemination of scientific research documents, whether they are published or not. The documents may come from teaching and research institutions in France or abroad, or from public or private research centers.
L'archive ouverte pluridisciplinaire HAL, est destinée au dépôt et à la diffusion de documents scientifiques de niveau recherche, publiés ou non, émanant des établissements d'enseignement et de recherche français ou étrangers, des laboratoires publics ou privés. 


\section{PARISSCHOQL OFECQNOMICS}

WORKING PAPER N $2015-03$

Joint Design of Emission Tax and Trading Systems

Bernard Caillaud

Gabrielle Demange

JEL Codes: D62, H23, Q54

Keywords: Climate policies, cap-and-trade mechanisms, emission tax, uncertainty

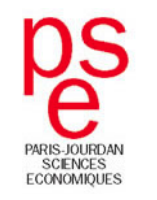




\title{
Joint Design of Emission Tax and Trading Systems*
}

\author{
Bernard Caillaud ${ }^{\dagger}$ Gabrielle Demange ${ }^{\ddagger}$
}

June 21, 2016

\begin{abstract}
This paper analyzes the joint design of fiscal and cap-and-trade instruments in climate policies under uncertainty. Whether the optimal mechanism is a mixed policy (with some firms subject to a tax and others to a cap-and-trade) or a uniform one (with all firms subject to the same instrument) depends on parameters reflecting preferences, production, and, most importantly, the stochastic structure of the shocks affecting the economy. This framework is then used to address the issue of the non-cooperative design of climate regulation systems in various areas worldwide under uncertainty. We characterize the resulting inefficiency, we show how the Pareto argument in favor of merging ETS of different regions is reinforced under uncertainty, and we discuss the non-cooperative design of mixed systems.
\end{abstract}

Keywords: Climate policies, cap-and-trade mechanisms, emission tax, uncertainty. Journal of Economic Literature Classification Number: D62, H23, Q54.

*This research is an outgrowth of a CEPREMAP 'Grand Project' that the authors developed in collaboration with J. Pouyet, who should receive some of the credit for this paper. The authors are grateful to CEPREMAP for its financial support. They have benefited from comments from two reviewers for this journal as well as from invited researchers at PSE and participants in the Conference 'Instruments to Curb Global Warming: Recent Developments,' co-organized with the CDC-Climat Recherche in Paris, the 5th Euro-African Conference in Finance and Economics in Agadir, and the World Congress of Environmental and Resource Economists in Istanbul, PEES in Paris.

${ }^{\dagger}$ Paris School of Economics - Ecole des Ponts ParisTech and CEPR. Corresponding author. Address: PSE, 48 boulevard Jourdan, 75014 Paris, France; Email: caillaud@pse.ens.fr.

${ }^{\ddagger}$ Paris School of Economics - EHESS and CEPR; E-mail: demange@pse.ens.fr. 


\section{Introduction}

United Nations have given up on trying to implement a unique carbon price, though economists promote it. ${ }^{1}$ The standard economic argument is efficiency, which requires all actors to face an identical marginal cost of pollution. A unique price in a given period can be achieved by imposing a tax worldwide or designing a global Emission Trading System (ETS), also called a cap-and trade system. But economists still discuss the relative merits of fiscal instruments compared to cap-and-trade mechanisms. ${ }^{2}$ In practice, the cap-andtrade approach seems more successful recently as various forms of ETS have been adopted in a few areas across the world, ${ }^{3}$ even in the non-ratifying US or in withdrawing Canada. ${ }^{4}$

Both systems produce very different outcomes due to the fluctuations economies are facing. Under a uniform ETS, i.e. an ETS that encompasses all activities, the shocks have no effect on emissions but induce fluctuations in the price and marginal cost of abatement, while under a uniform tax, i.e. a tax applying to all activities, they do not affect the marginal cost of abatement but generate a random volume of emissions. Restricting to a uniform system, one may prefer one or the other, as shown by Weitzman (1974). However, mixing the two systems could smooth the fluctuations in ETS prices and emission volumes, at the cost of departing from the doctrine of a unique carbon price. Could this be more efficient than a unique carbon price? The first objective of this paper is to provide an answer to this question based on a simple normative theoretical framework.

Observing the various emissions control systems that have emerged across the world, it is striking that these systems have not been elaborated more cooperatively and that their mechanisms exhibit many differences. ${ }^{5}$ Our result that a mixed system might be optimal worldwide is not incompatible with the emergence of different systems worldwide. Yet, the multiplicity of so many ETS has also raised the issue of linking various ETS as, from a standard economic point of view, linking two markets trading the same good is welfareimproving. Though, there are difficulties and resistance. A difficulty is well known: A Pareto improvement may call for transfers, which are not easy to implement. Also, non-economic features, such as the reliability of the trading system and of enforcement

\footnotetext{
${ }^{1}$ Before the COP21 conference, a large number of economists have signed a "call for an ambitious and credible climate agreement in Paris" in favor of commitments and a unique carbon price. See https://sites.google.com/a/chaireeconomieduclimat.org/tse-cec-joint-initiative/call . But the agreement reached in Paris hinges on a "pledge and review" mechanism in which countries set their own plans for carbon mitigation without any coordination nor any strong enforcement mechanism.

${ }^{2}$ See Guesnerie (2010) for a survey.

${ }^{3}$ Early starters are the Australian NSW (2003) and the EU ETS (2005). The EU ETS has now integrated Norway domestic emission trading, which started in 2005 too, and the UK ETS that started as early as 2002. The Swiss ETS ran 2008-2012, the Japan ETS for the Tokyo area started in 2010. The New Zealand ETS started in 2008.

${ }^{4}$ The Regional Greenhouse Gas Initiative (RGGI) started in 2009 and it caps emissions from power generation in ten north-eastern US states. Emissions trading in California and the Western Climate Initiative (WCI, a collective ETS agreed between 11 US states and Canadian provinces) are only a couple of years old.

${ }^{5}$ For a description of the existing or planned ETS as of 2013 see Talberg - Swoboda (2013).
} 
mechanisms are put forward by the EU (EU Report, 2008). Finally, difficulties arise due to the differences in the design of the existing or planned ETS. For example, the Australian White Paper (2008) was advocating a cap on the price of the permits for the planned Australian ETS, ${ }^{6}$ while the EU ETS has no such cap. The total amount of permits allocated to the firms submitted to an ETS and how this amount should evolve over time differ across areas. Also ETS may cover different sets of industries: e.g. the planned coverage of the Australian ETS was larger than the current EU ETS, which does not include transportation nor forestry. The second set of questions our paper addresses is therefore related to the uncoordinated design of mixed systems across various areas or countries around the world.

Our analysis relies on a static model that extends Weitzman (1974) by allowing for the possibility of the double control mechanism. The mechanism specifies which firms are submitted to a tax and which ones to an ETS, what we call the scope of the regulation, as well as the associated tax level and quota allocated to the ETS, what we call the policy. The mechanism is decided ex ante, before the realization of the shocks that affect the firms in the economy. This captures the fact that the regulatory framework cannot be contingent on the realization of shocks that hit constantly the economy. Yet, the firms' reaction to shocks should be taken into account when designing a regulatory framework. We characterize optimal (or equilibrium) policies for any scope and we explain how the stochastic structure of the shocks influences the optimal design of the scope of the regulatory framework. In particular, we analyze when it is preferable to adopt a uniform system, subjecting all firms to either a cap-and-trade mechanism or a tax on their emissions, or a mixed system in which some firms are regulated through a cap-andtrade mechanism and the others through a tax.

The basic forces at play are the following. If climate regulation could be made contingent on shocks, i.e. a first best scenario, abatement efforts should be determined so as to equalize marginal abatement cost across firms with the social marginal benefit of abatement: part of the aggregate shocks should then be absorbed through abatement at the firms' level and the coefficient of absorption would be larger, the less steep the aggregate marginal abatement cost curve, and the steeper the marginal abatement benefit curve.

In a regulatory framework characterized by a ETS sector and a taxed sector, the ETS sector absorbs all the shocks that impact it, which induces fluctuations in the corresponding marginal cost of abatement, while the taxed sector has a constant marginal cost of abatement but generates a random volume of emissions that reflects entirely the shocks that impact it. The optimal tax rate and ETS quota are determined so as to replicate the first best optimum in expected terms. Expected marginal abatement costs, that is the tax rate and the expected price on the ETS market, are equalized to their first best value; and expected net emissions are equalized to their first best optimal value as well.

\footnotetext{
${ }^{6}$ This ETS was supposed to start in 2015 but all climate legislation has been repealed in July 2014 due to a political swing.
} 
So, the precise definition of the scope of the regulatory framework, i.e. of the industries to be included in the ETS and of those to be taxed, only affects social welfare through the fluctuations due to the shocks. The optimal scope should then be designed so as to replicate as closely as possible the emissions fluctuations corresponding to the first best allocation, given that all fluctuations in emissions are generated by firms subject to the tax. A uniform ETS system, in which all firms are subject to the ETS regulation, eradicates all fluctuations while a uniform tax system induces all shocks to be passed on in emissions fluctuations. Comparing both systems amounts to assessing the relative slopes of the marginal abatement cost and benefit curves, as in Weitzman (1974). Improving on either system requires to analyze mixed systems, with a non-degenerate ETS sector and a non-degenerate taxed sector, and to calibrate the taxed sector so that the shocks that affect it are sufficiently correlated with the partially dampened aggregate shocks as required in the first best. But doing so creates a wedge between the marginal abatement cost of ETS firms and taxed firms: the carbon price is unique but only in expectation, not for all realized shocks. A mixed system is better than a uniform one when the social benefits drawn from emission fluctuations closer to the first best compensate for the loss due to the misallocation of abatement efforts across firms.

We then use this framework to address the issue of the non-cooperative design of climate regulation systems in various areas worldwide. We consider a world consisting of several areas, in which each area may use a double control mechanism. The noncooperative outcome is compared to the first best emission levels for the global economy and the corresponding inefficiency that results, i.e. excess in emissions worldwide, is precisely analyzed. Moreover, we analyze the proposal of linking ETS by considering a specific form of linking, that we call ETS merging. We extend the strong classical Pareto argument in favor of merging ETS: such a move benefits both areas, even without implementing transfers across them or changing the sovereign decisions with respect to the fiscal instruments, these benefits are reinforced in the uncertain framework we consider, and we precisely characterize them for each area. Finally, we analyze the situation where each country chooses a uniform system within its frontiers. First, for the same reasons that lead a mixed system to be optimal, it might be sub-optimal to have the countries choosing all a cap-and trade system or all a tax system. For example, it might be optimal to have that the US, say, impose a cap and trade mechanism and China a tax. We then analyze the non-cooperative choice of its uniform system by each country and show that the equilibrium exhibits too many countries under a tax system and too few ones under an ETS compared to the globally efficient architecture.

Our paper contributes to the long literature, pioneered by Weitzman (1974), on the use of price or quantity instruments in a framework characterized by uncertainty and asymmetric information about the shocks between the central authority and the economic agents. Within a framework of uncertainty on compliance costs, various ways of combining price and quantity instruments have been shown to provide welfare improvements: a three- 
part tariff (Roberts and Spence 1976), indexed or hybrid instruments allowing a variable quota (Pizer 2002 and Newell and Pizer 2008), joint use of price and quantity regulation in the context of multiple pollutants depending on their degree of substitutability or complementarity (Ambec and Coria 2013). Specifically, our paper is related to Mandell (2008) who analyzes the possibility of a mixed system in which some firms are subject to a price mechanism while others are subject to a quantity mechanism. Compared to our paper, Mandell (2008) focuses on the optimal scope in a more restricted framework in which there is a single common shock affecting all firms. He shows that a mixed system might be superior to a single uniform system. The first part of our analysis can be viewed as providing a generalization of this argument to general stochastic structures. Independently of our paper, Carlen and Hernandez (2013) consider a general structure of shocks (with imperfect correlation) but they restrict their analysis to two firms: in this setting, they obtain a simple version of our result on the minimal degree of positive correlation that is necessary to obtain an optimal mixed system. ${ }^{7}$

There is ample evidence that the volatility of the carbon price is large and various studies have analyzed empirically its determinants. In particular, Chevallier (2011) analyzes both the impact of industrial production and energy prices on the carbon market and confirms that both have an impact. ${ }^{8}$ This justifies our modeling choice, namely that the shocks to the economy play a crucial role in the determination of the optimal scope as they drive the volatility of both the ETS price and the emission of the firms submitted to the emissions tax.

The second part of our paper relates to the more recent but very active literature on the linkage of different climate regulation settings, most notably the linkage of different ETS. ${ }^{9}$ Most of this literature assumes away uncertainty and starts with an initial carbon policies in each country that possibly reflect the country's objective but are globally inefficient. ETS merging then can improve global welfare as it induces cost equalization among participating firms. But differences in objectives also lead countries to incorporate different cost containment or price control mechanisms in their local ETS and to take into account offset mechanisms such as CDM differently, which constitutes major obstacles to the merging of ETS. ${ }^{10}$ Compared to this literature, we adopt a simplified setting in

\footnotetext{
${ }^{7}$ In addition, they investigate the possibility of indexing the tax rate on the realized value of the ETS price: this possibility of indexing the tax rate on the realization of some uncertainty is potentially efficiency enhancing.

${ }^{8}$ More precisely, the author considers a Markov-switching VAR model with two states that is able to reproduce the boom - bust business cycle. Industrial production is found to impact positively (resp. negatively) the carbon market during periods of economic expansion (resp. recession), and the energy prices impact the Markov-switching model.

${ }^{9}$ This constitutes the common thread of the contributions of the special issue of Climate Policy (Volume 9, Issue 4, 2009); see also Ranson and Stavins (2012) and Metcalf and Weisbachy (2012).

${ }^{10}$ Jotzo and Betz (2009) focus on the difficulties raised by some important specifications of the Australian project, i.e. a price cap with unlimited access to international CDM. Linking the EU ETS scheme with such a scheme would have a large impact by effectively introducing a price cap for the global system and bypassing the European constraint on the use of offset mechanisms. Tuerk et al. (2009) and Sterk and Kruger (2009) argue similarly that very few direct or bilateral merging of ETS would be viable in
} 
which we rule out CDM and other offset mechanisms, and we consider that ETS markets are perfectly competitive with no control mechanisms. We, however, endogenize the (centralized and decentralized) determination of carbon policies by explicitly formalizing the role of uncertainty as the fundamental determinant in their design. Wood et al. (2013) and Heindl - Wood - Jotzo (2014) rely also on a stochastic model to assess various ways of linking carbon policies between two possibly asymmetric countries. Both papers take the situation of ETS merging as a benchmark. Wood et al. (2013) compares it with a situation in which one country is under a tax system and not a ETS and countries trade allowances, while Heindl - Wood - Jotzo (2014) compares it to the situation in which one country imposes an additional tax on its constituent firms, on top of the joint ETS requirement. By contrast, our paper provides an endogenous characterization of the mixed systems adopted in each country under separate regulation, taking explicitly into account the impact of shocks on welfare.

The plan of the paper is as follows. Section 2 provides a normative analysis of climate regulation from a worldwide perspective. Section 3 turns to the choices of several areas with two focuses: the incentives to merge ETS and the non-cooperative choices of regulatory instruments. Technical results are proved in two appendices, gathering results corresponding to Sections 2 and 3 .

\section{A normative analysis of climate policies}

We consider a global economy in which the production process generates a stochastic volume of emissions of a pollutant. Consumers care about the aggregate emissions volume. Firms may reduce emissions through costly abatement if they have incentives to do so. We restrict the analysis to two incentive instruments: an emission trading system (ETS) and a tax. Both instruments can be used simultaneously, with some activities subject to a tax and the others to an ETS.

The main questions we investigate in this section are: How to determine the firms covered by an ETS and those subject to a tax ? How to determine the quota of emissions allocated to the ETS firms and the tax rate imposed on non-ETS firms ? Our answers provide a normative approach to the design of climate policies from a worldwide perspective.

the short run. They also discuss why an indirect linkage emerges among carbon markets, through the recognition of CDM and other crediting mechanisms, and how it may help improve global efficiency. Anger (2008), in a similar vein, shows that significant and beneficial effects can arise from opening the Kyoto system, so far restricted to governments, to ETS firms, thereby effectively creating a world market. Flachsland et al. (2009) points out ETS market failures as well as strategic manipulation of national carbon policies as additional obstacles to viable merging of ETS. 


\subsection{The model}

We consider a standard model of a perfectly competitive economy under uncertainty in which firms emit pollutants that affect consumers. This framework relies on the following modeling assumptions. First, there is separability in terms of costs and welfare between the markets for the goods and the emissions volume: abatement decisions have no impact on the goods' equilibrium prices and traded quantities. Second, uncertainty is introduced in the form of shocks on gross (pre-abatement) levels of emissions; shocks on the abatement technology itself are ruled out. Finally, all cost and surplus functions are quadratic with respect to emissions volumes. This mild assumption can be viewed as an approximation of more general functional forms.

Firms. There are $n$ firms in the economy. Each firm $i, i \in N \equiv\{1,2, \ldots, n\}$, emits a volume of pollutant through its production activities when it does not make any abatement effort. Let $z_{i}+\epsilon_{i} \geq 0$ denote this volume. $z_{i}$ is assumed to be common knowledge in the economy; $\epsilon_{i}$ is known by firm $i$ at the time it decides how much to abate.

Firm $i$ has access to an abatement technology with linear marginal cost: ${ }^{11}$ abating $a_{i} \geq 0 \operatorname{costs} \frac{b_{i}}{2} a_{i}^{2}$ and reduces firm $i$ 's emissions down to a volume of net emissions $x_{i}=z_{i}+\epsilon_{i}-a_{i}$. The smaller $b_{i}$, the more elastic the firm's abatement decisions to a variation in the unit price of emissions, so that $\frac{1}{b_{i}}$ can be viewed as a measure of firm $i$ 's flexibility in abating.

At the time of the design of the regulatory instruments, the values of each $\epsilon_{i}$ are unknown, perceived as random. The random variable $\tilde{\epsilon}_{i}$ is referred to as firm $i$ 's shock. W.l.o.g. we suppose that $\widetilde{\epsilon}_{i}$ has zero mean so that $z_{i}$ is firm $i$ 's average emission volume. The structure of all shocks $\left(\widetilde{\epsilon}_{i}\right)$ in the economy plays an important role in the analysis and we provide below examples of the factors that shape this stochastic structure.

- A change in the emission by-product for a given input use in firm $i$, e.g. because of an innovation in its production process or technology, induces a direct change in the firm's gross emissions volume and consequently in the marginal abatement cost to achieve a given level of net emissions.

- A decrease in the prices of carbon-intensive inputs, e.g. a decrease in the oil prices, leads to an increase in the use of these inputs; this induces an increase in the gross emissions volumes of all firms using such an input and ultimately to an increase in the marginal abatement cost of these firms for a given level of net emissions; moreover these increases are correlated across all firms using this input.

- An increase in the demand for the goods in some industry increases the gross level of emissions through an increase in production, hence ultimately increases the marginal

\footnotetext{
${ }^{11} \mathrm{~A}$ linear term of the form $\mu_{i} a_{i}$ can be added without change in the analysis, up to a translation in the expressions for the emission volumes, prices and taxes.
} 
abatement cost for given net emissions for any firm in this industry; these effects are strongly correlated across the firms within the industry. For the same reason, a macroeconomic shock that affects all industries induces correlation across industries.

Firms are submitted to an emission tax or to an ETS, as will be described below. For the moment, let $\tau$ denote the unit cost firm $i$ is facing. Firm $i$ choosing to emit $x_{i}$ obtains net profits equal to:

$$
\Pi_{i}=\xi_{i}-\frac{b_{i}}{2}\left(z_{i}+\epsilon_{i}-x_{i}\right)^{2}-\tau x_{i},
$$

in which $\xi_{i}$ summarizes the net profits on the goods' markets, possibly random.

Given a set $S$ of firms, $S \subseteq N$, we use the following notation:

$$
z_{S}=\sum_{i \in S} z_{i}, x_{S}=\sum_{i \in S} x_{i}, \epsilon_{S}=\sum_{i \in S} \epsilon_{i} \text { and } \frac{1}{b_{S}}=\sum_{i \in S} \frac{1}{b_{i}} \text { with } b_{\emptyset}=0
$$

Let us interpret these expressions. $z_{S}$ is the total expected gross emissions volume for a group $S$ of firms, e.g. a sector or an industry, and $\epsilon_{S}$ is the total shock in gross emissions of this group. Thus $z_{S}+\epsilon_{S}$ corresponds to the gross emissions level of group $S$. Aggregating the abatement technologies of all firms in the group $S$, the abatement cost at the group's level is quadratic given by $\frac{b_{S}}{2} a^{2}$, since an abatement of $a$ units by the firms in $S$ is efficiently obtained by assigning shares to the firms in proportion of their flexibility, i.e. by assigning $\frac{b_{S}}{b_{i}} a$ to firm $i \cdot \frac{1}{b_{S}}$ then measures the flexibility at the group level.

Consumers surplus and social welfare. Consumers' loss due to emissions only depends on total emissions level $x_{N}$ and is also assumed to be quadratic. In terms of consumers' surplus, this gives:

$$
C S=\lambda-\nu x_{N}-\frac{A}{2} x_{N}^{2}
$$

where $\lambda$, possibly random, summarizes the surplus on the goods markets. This form for the consumers' surplus corresponds to a linearly increasing (social) marginal abatement benefit, equal to: $\nu+A x_{N}$. $A$ measures the slope of the marginal abatement benefit curve: it may be perceived as large if e.g. the current volume of emissions is such that catastrophic climatic consequences would follow a small increase in emissions given the current situation (threshold effect).

Finally, the revenues $R$ from the emission tax or the sales of permits are collected by a governmental agency. Assuming no cost of public funds, social welfare is given by $W=C S+R+\sum_{i \in N} \Pi_{i}$. Transfers within the economy are socially neutral. From (1) and (3), the expected welfare is (up to an additive term independent of the emission levels):

$$
\mathbb{E}\left\{-\sum_{i \in N} \frac{b_{i}}{2}\left(z_{i}+\epsilon_{i}-x_{i}\right)^{2}-\nu x_{N}-\frac{A}{2} x_{N}^{2}\right\}
$$


Optimality is defined with respect to this social welfare criterion.

The designer aims at maximizing this expression, taking into account the reaction of the firms and their knowledge of the shocks. We focus on situations in which there are restrictions on the regulatory tools that can be used : some firms are regulated by a cap-and-trade system and the others by an emissions tax, as we describe now.

Scope and policy. Let $N$ be partitioned in two subsets, $T$ and $Q$ with $N=T \cup Q$. Firms in $T$ are subject to the same tax rate $t$ per unit of net emissions. Firms in $Q$ are subject to a cap-and-trade mechanism in which a total amount $q$ of emissions is allowed; firms in $Q$ must remit as many allowances as units of net emissions they produce and they buy allowances on a (perfectly competitive) resale market that reallocates emission allowances across firms. ${ }^{12}$ Each firm is perfectly identified and allocated to one subset or the other at no cost, and its net emissions can be measured perfectly. ${ }^{13}(T, Q)$ is called the scope of the system and, given the scope, the policy consists in $(t, q)$. The system is said to be uniform when $T=N$ (all firms are submitted to a tax) or $Q=N$ (all firms are submitted to the ETS).

We use the term "firm" for simplicity. In practice, the assignment to an ETS is made at the level of the activity of a plant, so that a company may have only some plants submitted to an ETS. Furthermore, the assignment is not discretionary in the sense that all plants performing the same activity should be treated the same way, i.e. all assigned to the ETS or none. Our analysis is therefore better interpreted as applying to activities instead of firms; in that interpretation, the abatement cost of $i$ refers to the average cost of the plants performing activity $i$.

The scope and the policy are implemented before the shocks are realized. Then, after the scope and the policy are determined, uncertainty resolves and firms react by choosing emission levels so as to optimize their net profits and choose their positions on the ETS. The aim of this section is to investigate the scope and policy that maximize welfare.

The separability assumption. So far, we have assumed a strong separability between the goods markets and the environmental regulation, namely that gross emission levels and profits on the goods markets are not affected by the regulation instruments. Even in the absence of shocks, this assumption is debatable. An interaction surely exists, though there is no consensus about its magnitude, as testified by the vivid debate on the impact of the environmental tax policies on growth (see Stern, 2007, Nordhaus, 2007) or, in a shorter term perspective, on the labor market - the double dividend debate (Bovenberg

\footnotetext{
${ }^{12}$ We consider a single tax level and a single trading system. This is not a restrictive assumption as will be clear later on.

${ }^{13}$ So, we rule out the possibility that firms decide freely to which mechanism they are subject to, or that they arbitrage between the two mechanisms. In the EU ETS, firms are subject to the cap-and-trade mechanism based on observable characteristics and allowances cannot be used by firms outside the capand-trade to reduce their tax basis; they can of course behave as traders on the ETS market but our perfect competition assumption makes this possibility irrelevant.
} 
and Goulder, 1996). At a micro level, Martin, de Preux and Wagner (2014) find that a carbon tax has an impact on energy use, but do not find a significant effect on employment and production.

Our analysis of the optimal scope can be extended to a more general setting, as will become clear and made precise in the discussion following Proposition 1. According to this proposition, the optimal policy for a given scope is determined so that the expected ETS price is equal to the tax rate, both being equalized to their optimal counterpart absent uncertainty, which is independent of the scope. Consequently, our results extend to the situations where the direct effect of the environmental policy on production, prices and profits on the goods markets, as well as on gross emissions, is captured by mean values only, not by variations: the firms' perceived expected costs of emissions drive the mean value of production decisions. So, the critical assumption in our setting is that of a separation between the environmental policy and the variations - and not the expected values - of all production decisions, hence of gross emissions, around their means. ${ }^{14}$

First best allocation. To understand the various inefficiencies associated with the scope design, we first consider the first best optimal allocation. The first best optimal allocation maximizes the social welfare without any constraint assuming the shocks $\boldsymbol{\epsilon}=$ $\left(\epsilon_{1}, . ., \epsilon_{n}\right)$ to be known by the designer; it is obtained by maximizing welfare for each value of $\boldsymbol{\epsilon}$ separately. First best encompasses two conditions. First, cost efficiency, which requires that the cost of achieving the total emissions volume is minimized, i.e., the private marginal costs of abatement is equalized across firms ex post. Second, volume efficiency, which requires that the optimal total emissions volume emerges, i.e., that the common private marginal cost of abatement is equalized to the social marginal benefit of abatement. Formally, given $\boldsymbol{\epsilon}$, the first best emission volumes $x_{i}(\boldsymbol{\epsilon})$, for $i \in N$, satisfy: ${ }^{15}$

$$
\begin{aligned}
b_{i}\left(z_{i}+\epsilon_{i}-x_{i}(\boldsymbol{\epsilon})\right) & =m(\boldsymbol{\epsilon}) \text { for all } i \\
m(\boldsymbol{\epsilon}) & =\nu+A x_{N}(\boldsymbol{\epsilon})
\end{aligned}
$$

where $m(\boldsymbol{\epsilon})$ denotes the private marginal costs of abatement at the first best. Explicit expressions are easily obtained (the computation is detailed in Appendix A). Let $x_{i}^{*}=$ $x_{i}(\mathbf{0})$, for $i \in N$ and $m=m(\mathbf{0})$ denote the allocation and the marginal abatement cost obtained at first best when all shocks are equal to their mean (for $\epsilon_{i}=0$ for all $i$ ). We

\footnotetext{
${ }^{14} \mathrm{~A}$ more precise statement is relegated in the discussion following Proposition 1.

${ }^{15}$ We assume that all the $z_{i}$ are large enough compared to $\frac{\nu}{b_{i}}$ so that the first best allocation as well as those considered later on produce positive values for the net emissions. This avoids uninteresting corner solutions.
} 
obtain:

$$
\begin{aligned}
x_{i}^{*} & =z_{i}-\frac{m}{b_{i}} \text { for all } i \in N \text { with } m=\frac{b_{N}\left(\nu+A z_{N}\right)}{A+b_{N}} \\
x_{i}(\boldsymbol{\epsilon}) & =x_{i}^{*}+\epsilon_{i}-\frac{A b_{N}}{b_{i}\left(A+b_{N}\right)} \epsilon_{N} \text { for all } i \in N \text { and } m(\boldsymbol{\epsilon})=m+\frac{A b_{N}}{A+b_{N}} \epsilon_{N} .
\end{aligned}
$$

Since the allocation is linear in the shocks and the flexibility parameters, we obtain:

$$
x_{S}(\boldsymbol{\epsilon})=x_{S}^{*}+\epsilon_{S}-\frac{A b_{N}}{b_{S}\left(A+b_{N}\right)} \epsilon_{N} .
$$

In particular, the first best optimal aggregate volume of emissions is given by: $x_{N}(\boldsymbol{\epsilon})=$ $x_{N}^{*}+\frac{b_{N}}{A+b_{N}} \epsilon_{N}$. It is random and follows the aggregate shock on total gross emissions $\epsilon_{N}$ with a dampening coefficient $\frac{b_{N}}{A+b_{N}}$ smaller than 1 . This coefficient reflects the strength of decreasing returns in the aggregate abatement technology (the slope of the aggregate marginal abatement cost curve $b_{N}$ ) and the slope of the marginal abatement benefit curve $(A)$. When $b_{N}$ is small relative to $A$, variations in the level of net emissions induce small changes in the aggregate marginal abatement cost but large swings in the social marginal abatement benefit: therefore, preserving the equality between marginal abatement cost and benefit in the presence of shocks requires to absorb most of these shocks through abatement so that net emissions do not fluctuate much. Hence, the dampening coefficient is small. The stronger decreasing returns in abatement, the steeper marginal abatement costs and so, the larger the proportion of aggregate shocks on gross emissions that is passed on into net emissions so that as to maintain efficiency.

As is well known, in a certain world, the first best allocation $\left(x_{i}^{*}\right)$ can be reached easily either by imposing a tax on all firms $(T=N)$ or by organizing an $\operatorname{ETS}(Q=N)$ provided that the tax level or the level of quotas are well chosen so as to induce the optimal common marginal abatement cost $m$. More generally, the optimal allocation can be reached through any scope, since the policy can be chosen to induce the marginal cost $m$. This neutrality result no longer holds in the presence of uncertainty: the choice of the scope matters.

\subsection{Optimal tax and quota levels given a scope}

Given a scope $(T, Q)$, a policy $(t, q)$ determines how the emissions volume of the taxed sector and the transactions and prices within the ETS vary with the realized shocks. The optimal policy is chosen under correct anticipation of these reactions.

For a policy $(t, q)$, the amount emitted by a firm is given by $b_{i}\left(z_{i}+\epsilon_{i}-x_{i}\right)=\tau$, with $\tau=t$ in the taxed sector and $\tau=p$ in the ETS. Aggregating over firms, the amount emitted by the taxed sector and the price on the ETS, both random, are given by:

$$
\widetilde{x}_{T}=z_{T}-\frac{t}{b_{T}}+\widetilde{\epsilon}_{T} \text { and } \widetilde{p}=b_{Q}\left(z_{Q}+\widetilde{\epsilon}_{Q}-q\right) \text {. }
$$


The marginal abatement cost is $t$ for the firms in $T$ and $\widetilde{p}$ for firms in $Q$; so, expected marginal abatement costs are :

$$
t \text { for } i \in T \text { and } b_{Q}\left(z_{Q}-q\right) \text { for } i \in Q
$$

The first proposition characterizes the optimal policy $(t, q)$ for a given scope.

Proposition 1. Given a scope $(T, Q)$, the optimal policy sets the level of the tax on $T$ equal to $m$ and the quota on $Q$ equal to $x_{Q}^{*}$. For this policy, the expectation of the emissions volume by firms in $T$ and the expectation of the price level on the ETS are equal to their first best levels absent uncertainty, respectively $x_{T}^{*}$ and $m$.

Proof. The proof is given in Appendix A.

Proposition 1 shows that the optimal policy is set so as to equalize all firms' expected marginal abatement costs to $m$, the social marginal benefit of abatement at the optimal level absent uncertainty, and it yields in expected terms the first best optimal emission levels absent uncertainty. This is obtained by setting the optimal tax equal to $m$ and the quota on the ETS equal to the aggregate optimal emissions volume of the firms under the cap-and-trade absent uncertainty. At the optimal policy, the amount emitted by firms in the taxed sector can be written, using (10):

$$
\widetilde{x}_{T}=x_{T}^{*}+\widetilde{\epsilon}_{T}
$$

that is, the sum of all shocks on gross emissions in the taxed sector are passed on, without any dampening, into fluctuations in net emissions. By contrast, all shocks affecting the gross emissions in the ETS sector are completely wiped out by construction, but the price on the ETS is random and given by:

$$
\widetilde{p}=m+b_{Q} \tilde{\epsilon}_{Q}
$$

Let us come back on the possibility of modeling the impact of environmental policy on the goods markets. Let us first assume that firms subject to the ETS make their production decisions after observing their idiosyncratic shocks and on the basis of the expected ETS price, i.e. before they observe the realization of the ETS price; of course, they make their abatement and purchase of permits decisions after observing the ETS price. This assumption seems appropriate for all investment decisions and production plans that need be elaborated in advance and are less flexible than transactions on the ETS market.

Second, assume the impact of the policy instruments on the variations in gross emissions around their mean are negligible. Specifically, given a tax level or an expected permit price $\tau$, let $z_{i}(\tau)$ be $i$ 's gross emission level under certainty at the equilibrium of the market, i.e. accounting for the impact of $\tau$ on the goods market equilibrium. Shocks 
lead firms to adjust their production level resulting in a gross emission level, say $z_{i}\left(\tau, \eta_{i}\right)$ if $\eta_{i}$ denotes the production shock on firm $i$. We assume that we can neglect the impact of $\tau$ on the variation of gross emissions around the value without shock, i.e. $z(\tau, \eta)-z(\tau)$ is a random variable independent of $\tau$, which has a null expectation. Thus, $z(\tau, \eta)$ can be written as $z(\tau)+\epsilon$.

Third, welfare is quadratic in the choice variables. This assumption was already made on the emission costs and damage; hence it is extended to the surplus on the goods markets.

Under these three assumptions ${ }^{16}$ given a scope, the optimal policy (tax and quota) is the one that maximizes the welfare in the absence of shocks, as in Proposition 1. This implies that the tax is independent of the scope and the quota is set so that the expected permit price is equal to that optimal tax. The only difference with the previous analysis here is that this optimal policy accounts for the equilibrium effect on the goods markets. The analysis of the scope (see the next subsections) then carries through: since, whatever the scope, the tax and expected emission price are set to the optimal tax without uncertainty, the gross emission levels are independent of the scope.

\subsection{Optimal scope}

The first best allocation is characterized by the two conditions of cost efficiency and volume efficiency, as described by (5)-(6). Under uncertainty, whatever scope $(T, Q)$, there is little chance that both conditions are met for all realized shocks. Cost efficiency is not satisfied in a mixed system, as marginal costs are equalized either to the tax or to the (random) competitive market price on the ETS which typically differs from the tax (when $\widetilde{\epsilon}_{Q}$ is not equal to 0 ). Instead a uniform system is cost efficient. However a uniform system is not volume efficient in general. Recall that the ex post optimal volume of emissions is $x_{N}^{*}+\frac{b_{N}}{A+b_{N}} \widetilde{\epsilon}_{N}$. With a uniform ETS scope, the quantity is fixed and with a tax system, the aggregate volume is $x_{N}^{*}+\widetilde{\epsilon}_{N}$, which reflects one-for-one the aggregate shock on gross emissions and is therefore too sensitive to it. A mixed system, on the other hand, generates an aggregate (random) volume of emissions equal to: $x_{N}^{*}+\widetilde{\epsilon}_{T}$, which might better replicate the variations of the ex post optimal volume. ${ }^{17}$

To analyze further the strength of these two effects, we provide a decomposition of the loss in welfare due to the scope relative to the ex-post optimal allocation. Given a realization $\boldsymbol{\epsilon}$ of the shocks, let $W^{T, Q}(\boldsymbol{\epsilon})$ denote the welfare associated with a given scope $(T, Q)$, and its associated optimal policy as given by Proposition 1 , and let $W^{f b}(\boldsymbol{\epsilon})$ denote the optimal welfare level for the same realization of shocks, which is associated with the

\footnotetext{
${ }^{16}$ The key point in the argument is that marginal welfare with respect to a marginal variation in fiscal instruments is linear in the shocks. Its expectation is independent of the shocks and equal to the level in the absence of shocks (the certain equivalent).

${ }^{17}$ For a common shock $\theta$ on marginal abatement costs, i.e. if $b_{i} \epsilon_{i}=b_{S} \epsilon_{S}=\theta$ for each $i$ and $S$, the optimal quantity is emitted whatever $\theta$ if $b_{T}=A+b_{N}$. This is the insight of Mandell (2008).
} 
ex post optimal allocation. As proved in Appendix A, the welfare loss can be written as:

$$
W^{f b}(\boldsymbol{\epsilon})-W^{T, Q}(\boldsymbol{\epsilon})=\frac{1}{2}\left(A+b_{N}\right)\left(\frac{b_{N}}{A+b_{N}} \epsilon_{N}-\epsilon_{T}\right)^{2}+\frac{1}{2} b_{N} \frac{b_{Q}}{b_{T}} \epsilon_{Q}^{2} .
$$

The first term corresponds to the loss due to a sub-optimal aggregate emissions volume, as $\frac{b_{N}}{A+b_{N}} \epsilon_{N}-\epsilon_{T}$ corresponds to the difference between the optimal volume and the volume emitted under the scope $(T, Q)$. The second term corresponds to the loss due to the inefficient allocation of this total emissions volume across firms due to the differences in the marginal abatement cost across the taxed and the ETS sectors. The abatement of firms in $T$ is constant, equal to $\frac{t}{b_{T}}$, and that of firms in $Q$ is random, equal to $\frac{m}{b_{Q}}+\epsilon_{Q}$. To minimize the cost of abating $\epsilon_{Q}$, one should allocate it in proportion of the groups' flexibility levels, i.e. $T$ should abate $\frac{b_{N}}{b_{T}} \epsilon_{Q}$ and $Q$ should only abate $\frac{b_{N}}{b_{Q}} \epsilon_{Q}$. This explains why the loss is increasing in the ratio $\frac{b_{Q}}{b_{T}}$ and in the magnitude of the shocks.

Taking expectations over the distribution of the shocks, the overall expected loss can be written as:

$$
W^{f b}-W^{T, Q}=\frac{1}{2}\left(A+b_{N}\right) \mathbb{V}\left[\frac{b_{N}}{A+b_{N}} \widetilde{\epsilon}_{N}-\widetilde{\epsilon}_{T}\right]+\frac{1}{2} b_{N} \frac{b_{Q}}{b_{T}} \mathbb{V}\left[\widetilde{\epsilon}_{Q}\right] .
$$

This expression depends on the parameters determining the reactions of the firms and the consumers welfare, i.e. the slopes of the marginal abatement cost curves and of the marginal abatement benefit curve, and the shocks. The term affecting the expected marginal abatement costs and the expected marginal abatement benefit do not appear since we consider the optimal policy.

This expression is useful to understand the factors that favor a uniform system and those that favor a mixed system. Let us first determine the best uniform system. Applying expression (15) to uniform systems, the loss due to a misallocation of emissions across the sectors (the second term) is null (since $\mathbb{V}\left[\epsilon_{Q}\right]=0$ if $T=N$ and $\frac{1}{b_{T}}=0$ by convention if $Q=$ $N)$ and the loss corresponding to the aggregate volume is, up to the factor $\frac{1}{2\left(A+b_{N}\right)} \mathbb{V}\left[\widetilde{\epsilon}_{N}\right]$, equal to $A^{2}$ for a system with a uniform tax $(T=N)$ and to $b_{N}^{2}$ for a uniform ETS system $(T=\emptyset)$. Thus only the value of $A$ relative to $b_{N}$ matters and the best uniform system is determined, as in the case of a single firm treated in Weitzman (1974): if the slope of the marginal abatement benefit curve is steeper than that of the aggregate marginal abatement cost, making a mistake in the level of emissions is socially more costly than not minimizing the cost of abating, so that a uniform cap-and-trade system dominates, and conversely.

Now, comparing a mixed system with the best uniform system yields the following proposition.

Proposition 2. Denote $\mathcal{A} \equiv \min \left\{A, b_{N}\right\}$. The welfare loss associated with the scope 
$(T, Q)$ relative to the best uniform system is $\frac{1}{2} L(T, Q)$ where

$$
L(T, Q)=\left\{(A-\mathcal{A}) \mathbb{V}\left[\widetilde{\epsilon_{T}}\right]-2 \mathcal{A} \operatorname{cov}\left(\widetilde{\epsilon_{T}}, \widetilde{\epsilon_{Q}}\right)+\left(b_{N}-\mathcal{A}\right) \mathbb{V}\left[\widetilde{\epsilon_{Q}}\right]\right\}+b_{N} \frac{b_{Q}}{b_{T}} \mathbb{V}\left[\epsilon_{Q}\right]
$$

Thus a mixed system $(T, Q)$ is welfare improving if and only if $L(T, Q)$ is negative, or equivalently, if and only if

$$
\frac{b_{Q}}{b_{T}} \mathbb{V}\left[\widetilde{\epsilon}_{Q}\right]<2 \operatorname{cov}\left(\widetilde{\epsilon}_{T}, \widetilde{\epsilon}_{Q}\right)
$$

and

$$
1-\frac{2 \operatorname{cov}\left(\widetilde{\epsilon}_{T}, \widetilde{\epsilon}_{Q}\right)-\frac{b_{Q}}{b_{T}} \mathbb{V}\left[\widetilde{\epsilon}_{Q}\right]}{2 \operatorname{cov}\left(\widetilde{\epsilon}_{T}, \widetilde{\epsilon}_{Q}\right)+\mathbb{V}\left[\widetilde{\epsilon}_{Q}\right]}<\frac{A}{b_{N}}<1+\frac{2 \operatorname{cov}\left(\widetilde{\epsilon}_{T}, \widetilde{\epsilon}_{Q}\right)-\frac{b_{Q}}{b_{T}} \mathbb{V}\left[\widetilde{\epsilon}_{Q}\right]}{\mathbb{V}\left[\widetilde{\epsilon}_{T}\right]} .
$$

The loss is decomposed into the difference in the fluctuations between the best uniform system and $(T, Q)$ (the term within the large brackets in (16)) and the cost inefficiencies. Naturally, the optimal mixed system minimizes the loss. The exact determination of the optimal scope is however difficult, except in specific cases. ${ }^{18}$

Proposition 2 provides two necessary conditions, (17) and (18) for a mixed system to be welfare improving over the best uniform system and, together, these conditions are sufficient. If there exists a scope $(T, Q)$ that satisfies both conditions, then the optimal scope is mixed, although it might differ from the scope $(T, Q)$ that is tested.

The necessary condition (17) follows from (16) since the terms $(A-\mathcal{A}) \mathbb{V}\left[\widetilde{\epsilon}_{T}\right]$ and $\left(b_{N}-\mathcal{A}\right) \mathbb{V}\left[\tilde{\epsilon_{Q}}\right]$ are non-negative. When $A=b_{N}$ condition (18) is automatically satisfied. Recall that in that case, a uniform tax system and a uniform cap-and-trade system are equivalent. So, it is precisely when there is not a big welfare difference between two uniform systems that they are most likely to be dominated by a mixed system. ${ }^{19}$ When $A$ is larger (resp. smaller) than $b_{N}$, there are forces in favor of a tax system (resp. the ETS), which explains (18): a mixed scope improves upon a uniform one only if $\frac{A}{b_{N}}$ belongs to the interval around 1 defined by (18). Note that there is little evidence on how $A$ and $b_{N}$ differ worldwide as the aggregate marginal abatement benefits are far from being consensual even within the scientific community.

As we have seen, the loss due to the cost inefficiency of a mixed system is increasing in the ratio $\frac{b_{Q}}{b_{T}}$ and in the magnitude of the shocks, $\mathbb{V}\left[\widetilde{\epsilon}_{Q}\right]$. Condition (17) is a necessary condition for cost inefficiency of a mixed system to be smaller than the gain in volume efficiency generated by the fact that the mixed system tracks the fluctuations of the first best allocation better than the best uniform system, i.e., $\epsilon_{T}$ tracks $\frac{b_{N}}{b_{N}+A} \epsilon_{N}$. This is possible

\footnotetext{
${ }^{18}$ The choice variable, the scope, is a binary partition of $N$, which makes the optimization problem one over discrete variables, thereby preventing the use of differential techniques. For example, when all firms are symmetric, what matters is the number of firms under each regime, and the optimization problem is one over integers. Another issue is that the objective function might not to be well-behaved, even when relaxing the integer constraint.

${ }^{19}$ This does not mean that, in this case, the welfare gain from a mixed system is also small; indeed, when $A=b_{N}$ the welfare gain from a mixed system is proportional to $2 \operatorname{cov}\left(\widetilde{\epsilon}_{T}, \widetilde{\epsilon}_{Q}\right)-\frac{b_{Q}}{b_{T}} \mathbb{V}\left[\widetilde{\epsilon}_{Q}\right]$, which may be large.
} 
only if the shocks in the two sectors $T$ and $Q$ are positively correlated. The optimal scope is thus surely the best uniform system when the shocks affecting the firms are two-by-two negatively correlated or independent, but this is a rather implausible assumption. When some correlations are positive, the co-variance of the shocks between the two sectors must be strong enough.

Condition (17) is rather mild. A simple and plausible situation in which it holds is as follows: firm $i_{0}$ is affected by a low variance shock that is highly correlated with the high-variance shocks affecting (some) other firms in the economy. Then, condition (17) holds for $Q=\left\{i_{0}\right\}$ provided that firm $i_{0}$ 's flexibility is not too small compared to the other firms'. This does not necessarily mean that the optimal scope is such that $Q$ is equal to, or includes, $\left\{i_{0}\right\}$, but simply that a mixed system is optimal.

There is a fundamental asymmetry between $T$ and $Q$ in the criterion incorporated in (16), which is due to the external effects imposed by a firm on others in the ETS. Adding up a firm $i$ in the ETS increases the flexibility of the ETS sector and therefore its ability to absorb a given shock with less fluctuations in the ETS price. Moreover, if this additional firm in the ETS suffers no shock, whether it is assigned to the sector under the tax or to the ETS has no impact on the fluctuations in total emissions and overall, adding up firm $i$ in the ETS improves cost efficiency without affecting volume efficiency. Formally, this can be checked by computing the difference in our criterion when firm $i$, such that $\widetilde{\epsilon}_{i} \equiv 0$ is shifted from $T$ to $Q$ :

$$
L(T, Q)-L(T-i, Q+i)=b_{N}\left(\frac{b_{Q}}{b_{N-Q}}-\frac{b_{Q+i}}{b_{N-Q-i}}\right) \mathbb{V}\left[\widetilde{\epsilon}_{Q}\right]
$$

The difference is positive as $b_{Q}>b_{Q+i}$ and $b_{N-Q}<b_{N-Q-i}$, hence it is always worthshifting $i$ to $Q$. This argument leads to the following corollary, which contains a policy recommendation that is not usually invoked in the debate about the optimal scope design.

Corollary 1. If a mixed system is optimal, then the optimal ETS sector should incorporate all firms that are hit by small shocks.

Proposition 2 can be used to assess the performances of actual climate policies. The necessary condition for a mixed system to be better than the best uniform system is that it induces random net emissions (from the taxed sector) that are positively correlated with the variations of its ETS price. From (12), the fluctuations in the net emissions are equal to the shocks affecting the gross emissions of the firms that are subject to the tax, while from (13) the price fluctuations on the ETS market are positively correlated with the shocks affecting the gross emissions of the ETS firms. Analysts of existing emissions trading systems often consider that the price fluctuations of allowances are mainly driven by two factors: macroeconomic shocks and weather shocks. Macroeconomic shocks affect basically all sectors that emit GHG in a strongly correlated way. So, it is probably a good signal on the design of the European mixed system that the price of allowances is highly correlated with macroeconomic conditions, and therefore with shocks affecting 
gross emissions in non-ETS sectors (See Chevalier (2011)). By contrast, climatic shocks affect mostly the power and heat generation industries, as well as agriculture and farming in a milder way; they may however have only very limited impact on transportation and energy intensive industries. This suggests that a mixed system that would impose a capand-trade regulation only on the power and heat generation industries would probably be suboptimal since the co-variance term in (17) would be rather low, in particular if agriculture and farming were included in the ETS or if they represented a limited fraction of the economy. In other terms, in an area where agriculture and farming are important and are affected by strong weather fluctuations, e.g. perhaps as in Australia or North America, it may be preferable not to include them in a ETS mostly targeting power plants.

We close this section by illustrating Proposition 2 with two examples that allow us to provide a tighter characterization of the optimal scope. ${ }^{20}$

Example with a duplicated economy. Each activity $i \in I$ in the economy is duplicated into two sub-activities, $(i, 1)$ and $(i, 2)$, with $b_{(i, 1)}=b_{(i, 2)}$ and $\widetilde{\epsilon}_{(i, 1)}=\widetilde{\epsilon}_{(i, 2)}=\widetilde{\epsilon}_{i}$. Then, the scope $(T, Q)$, with $T=\{(i, 1), i \in I\}$ and $Q=\{(i, 2), i \in I\}$, satisfies (17).

Corollary 2. Assume the economy is duplicated, as described above. If $A$ and $b_{N}$ are not too different, a mixed system is optimal.

This example contains an important insight if we consider that the economy consists in several sectors populated by similar firms, active on the same markets and subject to the same shocks. Absent any technical or political constraints, it is beneficial in such a situation to split each sector so that a fraction of firms is under the cap-and-trade mechanism and the rest is subject to the tax: then, $\epsilon_{T}$ corresponds to a scaled-down version of the aggregate shock $\epsilon_{N}$, where the scale can be adjusted so as to equal $\frac{b_{N}}{A+b_{N}}$. Volume efficiency is maximized and dominates cost inefficiency. Such a mixed system would, however, have to treat similar firms differently, which raises obvious issues about acceptability.

Symmetric firms. All firms exhibit the same sensitivity to shocks, $b_{i}=b$ for all $i$, and the shocks $\epsilon_{i}$ have identical variance $\sigma^{2}$ and are two-by-two correlated with an identical correlation coefficient equal to $\rho$. The symmetry assumption implies $b_{S}=\frac{b}{|S|}$. The scope is summarized by $|T|$, the number of firms in $T$. The next corollary states the conditions under which a mix system is optimal.

Corollary 3. In the symmetric example, if $\frac{A}{b} \geq \rho$, a uniform ETS is optimal while if $\frac{A}{b} \leq \frac{\rho}{1-2 \rho+2 \rho n}$, a uniform tax system is optimal. Otherwise,

$$
\frac{\rho}{1-2 \rho+2 \rho n}<\frac{A}{b}<\rho
$$

\footnotetext{
${ }^{20}$ In the Appendix, we provide a third example involving a common shock on marginal abatement costs, as it is related to Mandell (2008).
} 
and a mixed system is optimal with a taxed sector whose size $|T|$ is an integer such that:

$$
\frac{b}{2 A}-\frac{1}{2 \rho} \leq|T| \leq \frac{b}{2 A}-\frac{1}{2 \rho}+1
$$

In the critical case in which the slope of the marginal abatement benefit curve is equal to the slope of the aggregate marginal abatement cost curve $\left(A=\frac{b}{n}\right)$, this proposition shows that the optimal scope necessarily incorporates an ETS (as $\frac{1}{n}>\frac{\rho}{1-2 \rho+2 \rho n}$ for any $\rho \in(0,1]$ and $n \geq 2)$ and that it is a mixed system if shocks are sufficiently positively correlated (i.e. if $\rho>\frac{1}{n}$ ).

Away from the critical case, the same conclusion applies when $A>\frac{b}{n}$, except with a higher threshold for the correlation across shocks (i.e. if $\rho>\frac{A}{b}$ ) and when $\frac{b}{2 n-1}<A<\frac{b}{n}$ except with a smaller, but still strictly positive threshold for the correlation across shocks. When, however, the slope of the marginal abatement benefit curve is very small compared to the slope of the aggregate marginal abatement cost curve, i.e. when $A<\frac{b}{2 n-1}$, the optimal scope is mixed if and only if the correlation coefficient falls within an interior interval, bounded away from independence and from perfect correlation. So, there is a rather large domain of parameter values within which a mixed system is optimal in this setting.

\subsection{The consumers and firms' viewpoint}

We consider here the point of view of the different actors in the economy on the design of the scope. It is immediate that consumers are better off when the scope is an uniform ETS: the expected volume of emissions is independent of the scope and consumers unambiguously dislike fluctuations in this volume of emissions. The firms' viewpoint is less obvious. Here, we ask the following question: given a scope, would a taxed firm rather be subject to cap-and trade, and conversely ? For that we compare firms' expected profits under both systems.

Given the realized $\epsilon_{i}$ simple computation yields that $i$ 's profit when it faces the cost $\tau$ (dropping the independent term $\left.\xi_{i}\right)$ is: $\Pi_{i}=\frac{\tau}{2 b_{i}}\left(\tau-2 b_{i}\left(z_{i}+\epsilon_{i}\right)\right.$ ). Taking expectations over the shocks, we obtain the expected profits at the optimal policy. For a firm $i$ subject to the tax, $\widetilde{\tau}=t=m$ and its expected profit is:

$$
\mathbb{E}\left[\widetilde{\Pi}_{i}\right]=\frac{m}{2 b_{i}}\left(m-2 b_{i} z_{i}\right) .
$$

For a firm $i$ subject to cap-and-trade, the cost of emission is equal to $\widetilde{\tau}=\widetilde{p}=m+b_{Q} \widetilde{\epsilon}_{Q}$, which gives the expected profit:

$$
\mathbb{E}\left[\widetilde{\Pi}_{i}\right]=\frac{m}{2 b_{i}}\left(m-2 b_{i} z_{i}\right)+\frac{1}{2 b_{i}} \operatorname{cov}\left(b_{Q} \widetilde{\epsilon}_{Q}, b_{Q} \widetilde{\epsilon}_{Q}-2 b_{i} \widetilde{\epsilon}_{i}\right) .
$$

The profit for a firm under tax is independent of the scope, as there is no external effect 
across firms and furthermore the tax level stays equal to $m$ whatever the scope. Instead, the profit for a firm under cap-and-trade depends on the scope as the price depends on the firms under the ETS.

Consider a firm $i$ under the ETS. We assume that $i$ compares its current profit to the profit it would achieve under the tax. ${ }^{21}$ For $i$ in $Q, i$ 's profit is larger under the cap-andtrade than under the tax if and only if the co-variance term in (21) is positive. The next proposition shows that firms tend to prefer being subject to a tax than to be included in the cap-and-trade mechanism.

Proposition 3. If $\mathbb{V}\left[\widetilde{\epsilon}_{Q}\right]>0$, then at least one firm in $Q$ would prefer to be subject to the tax, and this is the case for all firms when (a) the shock is common to all firms' marginal abatement costs, or (b) firms are symmetric: the shocks have identical variance and correlation and all flexibility parameters are identical $\left(b_{i}=b\right.$ for all $\left.i\right)$.

Proof. The proof is given in Appendix A.

From a political economy perspective, we can therefore expect that firms will likely be opposed to the design of a cap-and-trade mechanism and that those subject to it will actively lobby so that the system be abandoned or they be taken out of it.

Finally the comparison of the firms' profits in the two sectors holds more generally in the case of an emissions target. More precisely, given that expected marginal abatement costs are equalized across the two sectors, i.e. $b_{Q}\left(z_{Q}-q\right)=t$, the firms' profits are given by the same type of expressions as in (20) and (21), where the constant term is modified but still identical across sectors, so that the comparison of the profits only relies on the variance terms.

\section{Climate policies in a world with several areas}

A prominent issue in the design of a worldwide regulation of GHG emissions is that various countries around the world, or more generally various areas, have chosen their modes of regulation separately. Inefficiencies naturally arise from the non-cooperative design of climate policies in the absence of uncertainty. One route that has been discussed is to link the existing ETS of different areas, while preserving sovereignty of each area (country). We first provide a simple and quantitative assessment of a proposal to merge the various ETS across areas : such an agreement constitutes a clear improvement over the non-cooperative benchmark and it is easily implementable as it requires no compensatory transfers. We then discuss how the decentralized design of scopes matters: it does not

\footnotetext{
${ }^{21}$ For a firm under tax, $i$ in $T$, the reverse criteria says that $i$ compares its current profit to the profit it would achieve if it joined the ETS, when $Q \cup\{i\}$ is the ETS sector. This means that the firm accounts for its impact on the price of the ETS. An alternative assumption would be that the firm computes its profit under the observed price, when $Q$ is the ETS sector. The difference is likely to be negligible for most firms but not for a large electricity firm like EDF or for an industry.
} 
impact expected emissions but it affects welfare through the shocks and we analyze the interaction among areas at this scope design stage.

Consider a world consisting of several areas indexed by $\alpha \in \mathcal{W} ; N^{\alpha}$ denotes the set of firms in area $\alpha, N=\cup_{\alpha \in \mathcal{W}} N^{\alpha}$ and $N-N^{\alpha}=\cup_{\beta \in \mathcal{W}, \beta \neq \alpha} N^{\beta}$. Consumers are concerned with the worldwide emissions volume $x_{N}$. Consumers' surplus in area $\alpha$ is:

$$
C S^{\alpha}\left(x_{N}\right)=\lambda^{\alpha}-\nu^{\alpha} x_{N}-\frac{A^{\alpha}}{2} x_{N}^{2}
$$

So, consumers' surplus worldwide is given as before in (3) with $\nu=\sum_{\alpha \in \mathcal{W}} \nu^{\alpha}, \lambda=$ $\sum_{\alpha \in \mathcal{W}} \lambda^{\alpha}$ and $A=\sum_{\alpha \in \mathcal{W}} A^{\alpha}$. Let $m^{\alpha}\left(x_{N}\right)=\nu^{\alpha}+A^{\alpha} x_{N}$ denote the local marginal abatement benefit in area $\alpha$ for a global volume of emissions equal to $x_{N}$.

As in the previous section, the first best is not implementable because the regulation is designed before the realization of the shocks. When the scopes in each area are given, $\left(T^{\alpha}, Q^{\alpha}\right)$ for area $\alpha \in \mathcal{W}$, and climate policies are chosen by the world planner, a simple adaptation of Proposition 1 shows that these policies consist in fixing identical tax rates equal to the common marginal abatement cost at the worldwide optimum absent uncertainty. Specifically, let $x_{i}^{*}$ for $i \in N$ denote the firms' optimal volumes of emissions absent uncertainty and $m=\sum_{\alpha \in \mathcal{W}} m^{\alpha}\left(x_{N}^{*}\right)$ denote their common marginal abatement cost at this optimum, which is equal to the worldwide marginal abatement benefit. ${ }^{22}$ Optimal policies require for any area $\alpha \in \mathcal{W}: t^{\alpha}=m$ and quotas equal to the optimal emissions volumes absent uncertainty $q^{\alpha}=x_{Q^{\alpha}}^{*}$. Alternatively, these climate policy choices would be the ones made under a cooperative scenario across areas, for the given scopes. The following section instead considers a non-cooperative scenario.

\subsection{Decentralized choice of climate policies and merging of ETS}

We consider here the merging of ETS, keeping the other parameters of the climate policies - taxes and scopes - unchanged in each area.

The questions we investigate in the next proposition are: (1) Does the merging of ETS induce an increase in welfare in each area and can we characterize this welfare increase (without making specific assumptions on the policies) ? (2) Does the merger induce a change in the policies, given the existing scopes? In that purpose, we characterize the policy choices in the Nash equilibrium of the game in which all areas choose independently and simultaneously their climate policies, given the existing scopes, and we consider the effects of the merging.

Let us describe more precisely the type of merging and game we consider.

The merging of ETS results in a unique ETS for which the participants are all the firms initially subject to the ETS of their respective areas and the global quota is equal to the sum of the quotas in the areas: $Q=\cup_{\alpha \in \mathcal{W}} Q^{\alpha}$ and $q=\sum_{\alpha \in \mathcal{W}} q^{\alpha}$. We also assume

\footnotetext{
${ }^{22}$ Note that at the global optimum without uncertainty, the local marginal abatement benefit in area $\alpha$ is lower than its global value, i.e. $m^{\alpha}\left(x_{N}^{*}\right)<m$, because $m=\sum_{\alpha \in \mathcal{W}} m^{\alpha}\left(x_{N}^{*}\right)$.
} 
that the revenues from the sale of the permits are allocated to each area proportionally to its original quota.

Let us now consider the non-cooperative choice of policies. Suppose that, in each area $\alpha \in \mathcal{W}$, the scopes $\left(T^{\alpha}, Q^{\alpha}\right)$ are given and consider the game in which local policy designers choose their policies $\left(t^{\alpha}, q^{\alpha}\right)$ simultaneously and non-cooperatively, taking into account expected local welfare. Given scope $\left(T^{\alpha}, Q^{\alpha}\right)$ and policy $\left(t^{\alpha}, q^{\alpha}\right)$ in area $\alpha \in \mathcal{W}$, we let $\widetilde{\Pi}_{i}$ denote firm $i$ 's profit and $\widetilde{p}^{\alpha}$ the ETS price in area $\alpha$, so that the expected welfare in area $\alpha$, i.e. area $\alpha$ 's objectives in this game, can be written as:

$$
\mathbb{E}\left[C S^{\alpha}\left(\widetilde{x}_{N}\right)+\sum_{i \in N^{\alpha}} \widetilde{\Pi}_{i}+\widetilde{p}^{\alpha} q^{\alpha}+t^{\alpha} \sum_{i \in T^{\alpha}} \widetilde{x}_{i}\right]
$$

To study the Nash equilibria, following a similar approach as in Section 2, let us first consider the game absent uncertainty, i.e. assuming that $\boldsymbol{\epsilon} \equiv \mathbf{0}$. In that game, the scopes do not matter, and areas choose simultaneously the emissions volumes of their own firms, $i \in N^{\alpha}$ for $\alpha$, so as to maximize its local welfare, i.e. the welfare of the entities in the area. Let $x_{i}^{e}$, for all $i \in N$, denote the volumes of emissions in a Nash equilibrium of this game. These volumes of emissions (uniquely) solve the following system of equations:

$$
b_{i}\left(z_{i}+\epsilon_{i}-x_{i}^{e}\right)=\nu^{\alpha}+A^{\alpha} x_{N}^{e}=m^{\alpha}\left(x_{N}^{e}\right)
$$

for all $\alpha$ and $i \in N^{\alpha}$. Using these conditions, Appendix B shows that the global volume of emissions in the Nash equilibrium absent uncertainty satisfies:

$$
x_{N}^{e}=x_{N}^{*}+\frac{\sum_{(\beta, \gamma) \in \mathcal{W}^{2}, \beta \neq \gamma}\left[\frac{m^{\beta}\left(x_{N}^{*}\right)}{b_{N} \gamma}\right]}{1+\sum_{\beta \in \mathcal{W}}\left[\frac{A^{\beta}}{b_{N \beta}}\right]} .
$$

So, absent uncertainty, the Nash equilibrium allocation results in an excess in the global emissions volume compared to the worldwide social optimum, i.e. $x_{N}^{e}>x_{N}^{*}$, and the value of the firms' common marginal abatement costs and of the local marginal abatement benefits is larger than under the worldwide optimum for each area: $m^{\alpha}\left(x_{N}^{e}\right)>m^{\alpha}\left(x_{N}^{*}\right)$, for all $\alpha \in \mathcal{W} .^{23}$

We are now ready to answer the questions (1) and (2) related to merging and noncooperative behaviors.

Proposition 4. 1. Fix the scopes $\left(T^{\alpha}, Q^{\alpha}\right)$ and the policies $\left(t^{\alpha}, q^{\alpha}\right)$ in each area $\alpha \in$ $\mathcal{W}$. Then, merging the ETS systems (weakly) increases each area $\alpha$ 's local welfare for any realization of the shocks and yields each area in expectations a gain proportional to $\mathbb{E}\left[\left(\widetilde{p}^{\alpha}-\widetilde{p}\right)^{2}\right]$.

\footnotetext{
${ }^{23}$ Though there is an overall excess in emissions at the Nash equilibrium, it might not be the case for each area. It is possible that one area chooses a more stringent climate policy than what is worldwide optimal to 'compensate' for the lax policy of other areas.
} 
2. Fix the scopes $\left(T^{\alpha}, Q^{\alpha}\right)$ in each area $\alpha \in \mathcal{W}$. The climate policies at the noncooperative equilibrium are given by:

$$
t^{\alpha}=m^{\alpha}\left(x_{N}^{e}\right) \text { and } q^{\alpha}=x_{Q^{\alpha}}^{e}
$$

and they lead to the same expected volume of emissions as the Nash equilibrium absent uncertainty. Moreover if, starting from the equilibrium, ETS are merged, the same tax rates form an equilibrium in the game where areas can only choose their tax rates.

Proof. See Appendix B.

Point 1 of the proposition asserts that merging ETS is necessarily beneficial for each area, even in the absence of compensatory transfers across areas, assuming that the revenues from the sale of the permits are allocated to each area proportionally to its original quota. The argument is simple. First, under our scenario, the merging of the ETS has an effect on the firms under ETS and the revenues from the sales of the permits, but not on the taxed sectors nor the fiscal revenues from the emissions taxes and the consumers' welfare in each area. This is due to the fact that the volume of emissions of the taxed firms is identical under separate or merged ETS, which results in identical total volume of emissions as well. Hence the consumers' expected surplus in each area are also identical as they depend only upon the aggregate emissions volume. The comparison between the two situations (separate ETS or merged ETS) thus boils down to the comparison of the sum of expected profits of the firms under ETS and the revenues from the sales of the permits across the two situations.

Second, we show that, for each realized shocks, the sum of the expected profits of the firms under ETS and the revenues from the sales of the permits in an area increases (resp. is unchanged) provided the price on the merged ETS differs from the separate ETS price (resp. is equal). For given shocks $\boldsymbol{\epsilon}$, let $x_{i}^{\alpha}=z_{i}+\epsilon_{i}-\frac{p^{\alpha}}{b_{i}}$ and $x_{i}=z_{i}+\epsilon_{i}-\frac{p}{b_{i}}$ denote firm $i$ 's chosen emissions for a firm in area $\alpha$, under separate and merged ETS respectively, and let $\Pi_{i}^{\alpha}(\boldsymbol{\epsilon})=\xi_{i}-\frac{b_{i}}{2}\left(z_{i}+\epsilon_{i}-x_{i}^{\alpha}\right)^{2}-p^{\alpha} x_{i}^{\alpha}$ and $\Pi_{i}(\boldsymbol{\epsilon})=\xi_{i}-\frac{b_{i}}{2}\left(z_{i}+\epsilon_{i}-x_{i}\right)^{2}-p x_{i}$ the corresponding profits attained in each situation. Since $x_{i}$ maximizes firm $i$ 's profit when facing price $p$, the following must be true:

$$
\Pi_{i}(\boldsymbol{\epsilon}) \geq \xi_{i}-\frac{b_{i}}{2}\left(z_{i}+\epsilon_{i}-x_{i}^{\alpha}\right)^{2}-p x_{i}^{\alpha}=\Pi_{i}^{\alpha}(\boldsymbol{\epsilon})+\left(p^{\alpha}-p\right) x_{i}^{\alpha} .
$$

Summing over $i \in Q^{\alpha}$ and assuming all permits are sold under the initial situation, i.e. 
assuming that $\sum_{i \in Q^{\alpha}} x_{i}^{\alpha}=q^{\alpha}$, it follows: ${ }^{24}$

$$
\sum_{i \in Q^{\alpha}} \Pi_{i}(\boldsymbol{\epsilon})+p q^{\alpha} \geq \sum_{i \in Q^{\alpha}} \Pi_{i}^{\alpha}(\boldsymbol{\epsilon})+p^{\alpha} q^{\alpha}
$$

with a strict inequality if $p$ is different from $p^{\alpha}$. The left and right hand sides of the inequality are respectively the sum of the profits of the firms in $Q^{\alpha}$ plus the revenues from the sale of the permits for the regulation agency in $\alpha$ under merged ETS and under separate ETS respectively: when $p<p^{\alpha}$ the increase in firms' profits more than compensates the decrease in the ETS revenues (because of firms' adjustment) and when $p>p^{\alpha}$, the decrease in firms' profits is more than compensated by the increase in the ETS revenues.

Appendix B further shows that the gains from merging ETS at the level of an area is proportional to the expectation of the square of the difference in prices effective in this area between the situation with separate ETS and that with merged ETS, which can be decomposed into two non-negative terms. The first term is the square of the difference between expected ETS prices in both situations, $\left(\mathbb{E}\left[\widetilde{p}^{\alpha}\right]-\mathbb{E}[\widetilde{p}]\right)^{2}$. Unsurprisingly, the larger the difference in the expected prices between the two situations, the larger the increase in overall profits due to the equalization of marginal costs on average. The second term is the variance of the difference between $b_{Q^{\alpha}} \widetilde{\epsilon}_{Q^{\alpha}}$ and $b_{Q} \widetilde{\epsilon}_{Q}$, that is: $\mathbb{V}\left[b_{Q^{\alpha}} \widetilde{\epsilon}_{Q^{\alpha}}-b_{Q} \widetilde{\epsilon}_{Q}\right]$. Merging ETS therefore induces no additional welfare gain in the presence of shocks if all firms in $Q$ are subject to a common shock on their marginal abatement cost. But as soon as this is not the case, merging ETS induces a strictly positive additional welfare gain, compared to the case without uncertainty, which is due to a strict improvement in the absorption of shocks, and this gain increases when the global ETS incorporates firms that are subject to shocks on their marginal abatement costs that are less correlated with the shocks affecting firms in the ETS of area $\alpha$.

Point 2 of Proposition 4 considers the non-cooperative policy choices. According to (23), the marginal abatement costs in the taxed sector are equalized to the expected marginal abatement benefit in area $\alpha$ and the expected price in the ETS is equalized to the tax rate; so, expected marginal abatement costs are equalized across firms within each area. From (22), the expected aggregate emissions volume is independent of uncertainty and of the scopes fixed in each area. As a result, the tax rates are independent of the scopes, hence the expected marginal abatement costs and the expected emissions volume within each area as well.

It follows from this discussion that the policy choices produce the same outcome in expected terms as the ones that would be chosen at the Nash equilibrium in the absence of uncertainty. Inefficiencies associated to this Nash equilibrium come from two channels: first, there is no reason for the equalization of the marginal abatement costs or benefits

\footnotetext{
${ }^{24}$ One cannot exclude $\sum_{i \in Q^{\alpha}} x_{i}^{\alpha}<q^{\alpha}$. In that case, the price equilibrium $p^{\alpha}$ is null so the revenues before the merger are null; as for the revenues after the ETS are merged, they are also equal to $p q^{\alpha}$ : either $p>0$ and all permits are sold or $p=0$. Hence the same inequality holds.
} 
across areas, as the levels $t^{\alpha}$ are likely to differ across areas; and second, there is an excess of emissions compared to the worldwide optimum (even if areas are all symmetric). This excess comes from the fact that each area is concerned with the surplus of its own consumers only, as reflected by the expression for the tax levels in (23).

The merger considered in Point 1 of Proposition 4 assumes that the tax levels are fixed and equal across the two scenarios. According to the last statement of Point 2, this is a valid assumption when the initial tax levels form an equilibrium: Starting from the Nash equilibrium, merging ETS does not induce areas to change their specific tax rates thereafter. The argument is as follows. The best response tax level in an area depends on the taxes chosen by the other areas and the overall emission level. Since the merger does not affect the emission volumes of the ETS sectors, the best responses are unaffected by the merging, hence the Nash equilibrium is unchanged as well. Note that once ETS are merged, cost efficiency does not hold anymore within an area: the local tax rate, which is initially equal to the expected price within the area, will in general differ from the expected price on the merged ETS. ${ }^{25}$

Overall, Proposition 4 suggests that simply merging ETS is an easy way for separate areas to improve on global efficiency without too much adjustments. Merging ETS benefits all areas, hence it does not make it necessary to implement compensatory transfers across areas. Furthermore, it does not lead areas to change their tax rates when they are initially in equilibrium. Merging ETS is thus a simple and beneficial mechanism, which should be consensual. Some firms, however, may object to the merging of ETS. As we have seen, the firms' profits decrease in an area for the shocks under which the merged ETS price is higher than the separate ETS price. This occurs in particular when the separate ETS price is lower than that of all other areas. This is more likely to occur, the lower the expected price relative to that of others. ${ }^{26}$ When the policies are equilibrium policies, the expected prices are equal to the taxes in each area. Thus, the firms in the area in which the tax level is the lowest may object a merger of ETS.

\subsection{Decentralized choice of uniform scopes}

In the previous analysis, we have taken the scopes in each area as given. We now consider the non-cooperative choice of scopes in the specific setting in which each country chooses a uniform system locally. Before, let us discuss the optimal determination of scopes in an international system and the related issue of a 'unique carbon price' in that setting.

Suppose that the US, say, impose a cap and trade mechanism and China a tax. We might think that this uncoordinated international system is not as efficient as if both

\footnotetext{
${ }^{25}$ As an additional comment, note that we do not compare the Nash equilibrium with separate systems with the equilibrium of a game in which areas choose their policies anticipating that quotas will be merged; such a situation in analyzed in Helm (2003).

${ }^{26} \mathrm{An}$ increase in the expected price does not necessarily imply a decrease in expected profit, as there may be an additional benefit to the merger due to a decrease in the price volatility.
} 
areas had the same system. Drawing on Section 2.3 the analysis suggests, however, that it may not be worse than a unified system on an efficiency ground.

When the firms within a country are all under the same uniform system, they can be aggregated into a single one with a flexibility parameter equal to the aggregate one and a shock equal to the aggregate shock within the countries (thanks to the quadratic setting). Proposition 2 then applies to the universe in which each $i$ represents a country. When a mixed system is optimal, having some countries under a tax system and the others within a ETS is better than a unified system. It is important to stress that this result holds only under strong conditions on the policies: the policies must be 'globally' optimal, meaning that they maximize the expected social welfare worldwide given the scopes, and this implies a unique carbon price 'in expectation', equal to the worldwide marginal social cost. In our setting, this requires an identical tax level across the countries choosing the tax system and a unique ETS with an adequate quota resulting in an expected price equal to the common tax. However, this can be obtained only under coordination in the policy choices, a strong assumption.

We consider instead a non-cooperative setting in which each country chooses a uniform system and a policy that apply to its residents. The policies are chosen non-cooperatively, as in point 2 of Proposition 4, and therefore only depend on other countries' choices through their (random) global emissions level. The scopes matter only because there are shocks; their determination does not impact the average emissions volume but it does impact the random component of the emissions, hence the expected welfare at the local level of each area.

Let us determine the best uniform system of a country $\alpha$ facing the emissions volume of the rest of the world, denoted by $y+\widetilde{\eta}$. Country $\alpha$ 's best response depends on the difference in welfare between a uniform cap-and-trade system and a tax system. This difference is equal to:

$$
\frac{\left(A^{\alpha}-b_{N^{\alpha}}\right)}{2} \mathbb{V}\left[\widetilde{\epsilon}_{N^{\alpha}}\right]+A^{\alpha} \operatorname{cov}\left(\widetilde{\epsilon}_{N^{\alpha}}, \widetilde{\eta}\right) .
$$

This result can be derived as follows. As shown in Proposition 4, the optimal policies under a tax or a cap-and-trade systems have the same impact on the expected marginal abatement costs and emission volumes and react to $y$ similarly. As a result, the welfare in the two systems differ only through their impact on the variance of the shocks. The impact due to the variations in the abatement costs are respectively null under a tax and equal to $-\frac{b_{N \alpha}}{2} \mathbb{V}\left[\widetilde{\epsilon}_{N^{\alpha}}\right]$ under cap-and-trade; those due to the variations in emissions volume are respectively $-\frac{A^{\alpha}}{2} \mathbb{V}\left[\widetilde{\epsilon}_{N^{\alpha}}+\widetilde{\eta}\right]$ and $-\frac{A^{\alpha}}{2} \mathbb{V}[\widetilde{\eta}]$. Collecting terms, we obtain $(24)$ for the difference in welfare between the cap-and-trade and the tax systems.

Now, consider an equilibrium, in which each country chooses a best response. Let $\mathcal{T}$ represent the set of countries choosing a uniform tax system. From the viewpoint of country $\alpha$, we have $\widetilde{\eta}=\sum_{\beta \in \mathcal{T}, \beta \neq \alpha} \widetilde{\epsilon}_{N^{\beta}}$. Thus, from (24) the uniform cap-and-trade system is the best choice for $\alpha$ if 


$$
\left(A^{\alpha}-b_{N^{\alpha}}\right) \mathbb{V}\left[\widetilde{\epsilon}_{N^{\alpha}}\right]+2 A^{\alpha} \operatorname{cov}\left(\widetilde{\epsilon}_{N^{\alpha}}, \sum_{\beta \in \mathcal{T}, \beta \neq \alpha} \widetilde{\epsilon}_{N^{\beta}}\right) \geq 0
$$

The choice between the uniform systems relies on the comparison between the slopes of the local marginal abatement benefit curve and of the local marginal abatement cost curves and on the correlation between the variations in $\widetilde{\epsilon}_{N^{\alpha}}$ and the shocks $\sum_{\beta \in \mathcal{T}} \widetilde{\epsilon}_{N^{\beta}}$ in the areas that have chosen the tax system.

We characterize three effects. First, the country does not take into account the externalities that the variations of its emissions generate on other countries: the social cost is measured by $A^{\alpha}$ and not $A$. This effect tends to favor the tax system relative to the global optimum. Second, considerations of cost efficiency across countries do not enter into a country's choice, since taxes and ETS are separate per country: the adjustment parameter is measured by that in country $\alpha, b_{N^{\alpha}}$, and not by the smaller value $b_{N}$. This again tends to favor the tax system relative to the global optimum. Third, a positive correlation between the shocks increases the benefit of choosing the ETS: it reinforces the variations in the foreign emissions with the home ones if the tax system is chosen. The opposite effect occurs when the correlation is negative. In the sequel we assume the more plausible case of independent or positively correlated shocks. In that case the incentives for $\alpha$ to choose a uniform cap-and-trade system hence increase with the set $\mathcal{T}$.

From this discussion, it is unclear whether the non-cooperative choice results in too many or too few countries under a tax regime. It seems however that in general the two effects favoring the tax system should outweigh the correlation effect.

Indeed, in the independent case, a country $\alpha$ chooses a uniform cap-and-trade at equilibrium if $A^{\alpha}>b_{N^{\alpha}}$ and otherwise a tax system. This condition says that each country makes the same choice as if it were in isolation. But recall that a global capand-trade system is optimal over a global tax system only if $A>b_{N}$, which is a (much) weaker condition than $A^{\alpha}>b_{N^{\alpha}}$ since $A>A^{\alpha}$ and $b_{N}<b_{N^{\alpha}}$.

Only a strong enough correlation of the shocks across countries may reverse this effect. To evaluate further the effects, let us consider the case of $n$ symmetric countries: they have a common social cost $A^{\alpha}=\frac{A}{n}$, a common marginal abatement cost $b_{N^{\alpha}}=b$ and they are hit by shocks with identical variance $\sigma^{2}$ and with correlation coefficient $\rho>0$ : $\mathbb{V}\left[\widetilde{\epsilon}_{N^{\alpha}}\right]=\sigma^{2}$ for all $\alpha$, and $\operatorname{cov}\left(\widetilde{\epsilon}_{N^{\alpha}}, \widetilde{\epsilon}_{N^{\beta}}\right)=\rho \sigma^{2}$ for all $\alpha$ different from $\beta$. In this symmetric case, the incentives for $\alpha$ to choose a uniform cap-and-trade system simply depend on the sign of $\left(\frac{A}{n}-b\right)+2 A \rho \frac{n_{t}}{n}$ where $n_{t}$ is the number of countries other than $\alpha$ choosing a uniform tax system. The following proposition characterizes the equilibrium.

Proposition 5. Consider the symmetric case where countries choose a uniform system non-cooperatively. If $\frac{A}{b} \geq n$, all countries choose a uniform cap-and-trade at equilibrium and if $\frac{A}{b} \leq \frac{n}{1-2 \rho+2 \rho n}$, a tax system. Otherwise

$$
\frac{n}{1-2 \rho+2 \rho n}<\frac{A}{b}<n
$$


and the equilibrium number of countries choosing a cap-and-trade system is an integer such that:

$$
\frac{n}{\rho} \frac{b}{2 A}-\frac{1}{2 \rho} \leq|T| \leq \frac{n}{\rho} \frac{b}{2 A}-\frac{1}{2 \rho}+1 .
$$

Let us compare the Nash equilibrium with the optimal centralized choice, as is characterized by Corollary 3. In both situations, the country's expected marginal cost are approximately equal. In the centralized choice, optimality requires equality. In the Nash analysis, we know that the country's expected marginal cost are independent of its choice between a tax or a cap-and-trade system. By symmetry, up to integer differences, the expected marginal cost is thus equalized across all countries. The comparison between (26) and (19) shows that there are too many countries choosing the tax system in equilibrium (because $n>1 \geq \rho$ ).

\section{Conclusion}

This paper has proposed a normative analysis of the global design of environmental regulation in a world plagued with uncertainty, thereby providing a framework to understand when a mixed regulation, relying on both a cap-and-trade mechanism and a tax system, is optimal.

Adopting then a more realistic approach of non-cooperative design of local regulation by local authorities, the paper has analyzed a cooperative scenario corresponding to the merging of existing ETS, even with different scopes across areas. Focusing then on an entirely non-cooperative setting, the paper has characterized the inefficiencies involved and the impact of scopes, and it has investigated the excessive use of taxation instruments when local authorities decide non-cooperatively which system to use.

Our analysis relies on some key assumptions that have been discussed and that could be relaxed. The assumption of a linear-quadratic setting is obviously strong and it facilitates the characterization of optimal policies and welfare effects. In a general model, the Paretoimprovement obtained by merging ETS would probably cease to be true and only an aggregate (worldwide) welfare improvement would remain. The results still suggest that the issue of compensating transfers may not be critical in a smooth enough world for which the quadratic approximation is acceptable locally.

The assumption of separability of the environmental regulation and the goods markets has also been discussed and we have shown how to relax it. Still, some separability is necessary for our results. Reintroducing a richer interaction between the goods markets and the environmental regulation would invalidate our separate analysis of policy and scope and would surely forbid any Pareto argument for merging ETS. But again, if the interaction is limited, as seems to be the case in the data, the departure from our results should be limited.

Our results can also easily be amended to a setting in which the designer is subject to an additional constraint on its expected emissions. Indeed, areas that, as the European 
Union, have signed the Kyoto protocol have to meet an additional emissions target, which constrains their choice of an optimal scope and associated policies. Viewing such an emissions target as a strictly-enforced cap on (expected) emissions amounts to introducing the constraint: $\mathbb{E}\left[\widetilde{x}_{N}\right] \leq x_{N}^{\max }$. When $x_{N}^{\max }<x_{N}^{*}$, we can show that this additional constraint only modifies the deterministic parts of the net emissions and of the policy levels; however, the effects of the shocks on welfare are unchanged, so that the determination of the optimal scope is the same as before. ${ }^{27}$

There is a last dimension that we neglect in our paper, namely, the political economy considerations behind the design of environmental regulation. We have discussed briefly how firms may oppose a cap-and-trade system and we also have mentioned the distributive effects within each area of the merger of ETS. But a more systematic understanding of the political economy forces at play would certainly enrich our understanding of how climate regulation is shaped and if and how it can evolve as it is most likely that, in practice, the design of scopes in various areas is very much a political economy issue in which local firms and local lobbies influence the determination of the scope.

\footnotetext{
${ }^{27}$ From an analytic point of view, adding the constraint to the program modifies the objective by adding a linear term of the form $\lambda\left(x_{N}^{\max }-\mathbb{E}\left[\widetilde{x}_{N}\right]\right)$ for an appropriate multiplier $\lambda$. The result follows as linear terms have no impact in our analysis. Formally, this simply modifies the value of $m$ by substituting $\nu+\lambda$ to $\nu$.
} 


\section{References}

Ambec, S. and Coria, J. (2013): "Prices vs quantities with multiple pollutants," Journal of Environmental Economics and Management, 66(1), 123-140.

Anger, N. (2008): "Emissions trading beyond Europe: Linking schemes in a post-Kyoto world," Energy Economics, 30(4), 2028-2049.

Bovenberg, A. L., and Goulder, L. H. (1996): "Optimal environmental taxation in the presence of other taxes: general-equilibrium analyses", The American Economic Review, 86(4), 9851000 .

Carlen, B., and A. Hernandez (2013): "Indexing European CArbon Taxes to the EU ETS Permit Price - A Good Idea?", CTS Working Paper 2013-33, Centre for Transport Studies, Stockholm.

Chevallier, J. (2011): "A Model of Carbon Price Interactions with Macroeconomic and Energy Dynamics," Energy Economics, 33(6), 1295-1312.

Department of Climate Change, (2008): "Carbon Pollution Reduction Scheme: Australia's Low Pollution Future," White Paper, Commonwealth of Australia, Canberra.

Flachsland, C., Marschinski, R. and O. Edenhofer (2009): "To Link or not to Link: Benefits and Disadvantages of Linking Cap-and-Trade Systems", Climate Policy, 9(4), 358-372.

Guesnerie, R. (2010), Pour une politique climatique globale-Blocage et ouvertures, Opuscules du CEPREMAP, Editions Rue d'Ulm, Paris.

Heindl, P., P. Wood and F. Jotzo (2014): "Combining International Cap-and-Trade with National Carbon Taxes", CCEP working paper 1418, Australian National University.

Helm, C. (2003): "International Emissions Trading with Endogenous Allowance Choices," Journal of Public Economics, 87(12), 2737-2747.

Jotzo, F. and Betz, R. (2009): "Linking the Australian Emissions Trading Scheme," Research Reports 0914, Environmental Economics Research Hub, Crawford School of Public Policy, The Australian National University.

Mace, M. J., Miller, I., Schwarte, C., Anderson, J., Broekhoff, D., Bradley, R., Bowyer, C. and Heilmayr, R. (2008): "Analysis of the Legal and Organizational Issues Arising in Linking the EU Emissions Trading Scheme to Other Existing and Emerging Emissions Trading Schemes," Foundation for International Environmental Law and Development, London.

Martin, R., de Preux, L.B., and Wagner, U.J., (2014): "The Impact of a Carbon Tax on Manufacturing: Evidence from Microdata", Journal of Public Economics, 117, 1-14.

Metcalf, G.E. and Weisbachy, D. (2012): "Linking Policies When Tastes Differ: Global Climate Policy in a Heterogeneous World," Review of Environmental Economics and Policy, 6(1), 110-129. 
Mandell, S. (2008): "Optimal Mix of Emissions Taxes and Cap-and-Trade," Journal of Environmental Economics and Management, 56(2), 131-140.

Newell, R. G. and Pizer, W. A. (2008): "Indexed regulation," Journal of Environmental Economics and management, 56(3), 221-233.

Nordhaus, W., (2007): "A review of the 'Stern Review' on the Economics of Climate Change", Journal of Economic Literature, 45(3), 686-702.

Pizer, W. A. (2002): "Combining price and quantity controls to mitigate global climate change," Journal of public economics, 85(3), 409-434.

Ranson, M. and Stavins, R.N. (2012): "Post-Durban Climate Policy Architecture Based on Linkage of Cap-and-Trade Systems", Climate Change and Sustainable Development Series, 43.

Roberts, M. J. and Spence, M. (1976): "Effluent charges and licenses under uncertainty," Journal of Public Economics, 5(3), 193-208.

Sterk, W. and J. Kruger (2009): "Establishing a Transatlantic Carbn Market", Climate Policy, $9(4), 389-401$.

Stern, N., (2007), The Economics of Climate Change: The Stern Review, Cambridge University Press.

Talberg, A. and K. Swoboda (2013): "Emissions Trading Schemes around the World", background note, Parliament of Australia.

Tuerk, A., Mehling, M., Flachsland, C. and W. Sterk (2009): "Linking Carbon Markets: Concepts, Case Studies and Pathways", CLimate Policy, 9(4), 341-357.

Weitzman, M.L. (1974): "Prices vs. Quantities," The Review of Economic Studies, 41(4), 477491.

Wood, P., Heindl, P., Jotzo, F. and A. Loschel (2013): "Linking Price and Quantity Pollution Controls under Uncertainty", ZEW discussion paper 13-025. 


\section{Appendix A: Global normative analysis}

\section{Full information optimum.}

To avoid repetition we consider here the general case where there are shocks $\widetilde{\boldsymbol{\epsilon}}$ within an area and outside emissions given by $y+\widetilde{\eta}$. In the case of a worldwide planner, all the shocks are included in $\boldsymbol{\epsilon}$ and $y=0$.

When the planner has full information on the shocks realized in its area and the outside shock $\widetilde{\eta}$, he maximizes social welfare by choosing $x_{i}(\boldsymbol{\epsilon}, \eta)$, which in our quadratic setting is fully characterized by the FOC:

$$
\text { for any } i \in N, b_{i}\left(z_{i}+\epsilon_{i}-x_{i}\right)=\nu+A\left(x_{N}+y+\eta\right) \equiv m(\boldsymbol{\epsilon}, \eta) \text {. }
$$

Dividing by $b_{i}$ for each $i$, summing up over all $i \in N$, and gathering the terms in $x_{N}$, one gets:

$$
\begin{aligned}
x_{N}(\boldsymbol{\epsilon}, \eta) & =\frac{b_{N} z_{N}-(\nu+A(y+\eta))}{A+b_{N}}+\frac{b_{N}}{A+b_{N}} \epsilon_{N} \\
m(\boldsymbol{\epsilon}, \eta)=b_{N}\left(z_{N}+\epsilon_{N}-x_{N}(\boldsymbol{\epsilon}, \eta)\right) & =\frac{b_{N}\left(\nu+A\left(y+\eta+z_{N}\right)\right)}{A+b_{N}}+\frac{A b_{N}}{A+b_{N}} \epsilon_{N} \\
x_{i}(\boldsymbol{\epsilon}, \eta) & =z_{i}+\epsilon_{i}-\frac{m(\boldsymbol{\epsilon}, \eta)}{b_{i}} \text { for all } i \in N
\end{aligned}
$$

Let $y=0$ and $\eta \equiv 0$. Absent any uncertainty on $\boldsymbol{\epsilon}$, i.e. setting $\epsilon_{i} \equiv 0$ for all $i$, one obtains the characterization (7) with the value $m$ defined by $m=\frac{b_{N}\left(\nu+A z_{N}\right)}{A+b_{N}}$ and then, for any subset $S$ of $N, x_{S}^{*}=z_{S}-\frac{m}{b_{S}}$. Under uncertainty on $\boldsymbol{\epsilon}$, writing all variables as deviations from the same variables absent uncertainty, i.e. from the variables with a $*$, we obtain (8).

When $y>0$, the expressions are simply adjusted by constant terms, replacing $\nu$ by $\nu+A y$ in the quantities with a $*$, i.e. without uncertainty.

\section{Proof of Proposition 1.}

As above, we include the proof in the case of an outside emission $y+\eta$. For any $i \in T$, firm $i$ maximizes its profit net of the $\operatorname{tax}(\tau=t)$, i.e. it chooses $x_{i}$ such that $b_{i}\left(z_{i}+\epsilon_{i}-x_{i}\right)=t$ when the shock is $\epsilon_{i}$; then, $x_{T}=z_{T}-\frac{t}{b_{T}}+\epsilon_{T}$. For any $i \in Q$, firm $i$ maximizes its profit net of the cost of purchasing the $x_{i}$ permits $(\tau=p)$, and the market for permits clears at a perfectly competitive price $p$ given the realization of uncertainty $\boldsymbol{\epsilon}$ :

$$
\begin{aligned}
x_{i} & =z_{i}+\epsilon_{i}-\frac{p}{b_{i}}, \\
\sum_{i \in Q} x_{i} & =q .
\end{aligned}
$$


Summing up all $x_{i}$ for $i \in Q$, one gets: $p=b_{Q}\left(z_{Q}-q+\epsilon_{Q}\right)$. Moreover, aggregating over $Q$, the following holds: $z_{i}+\epsilon_{i}-x_{i}=\frac{b_{Q}}{b_{i}}\left(z_{Q}+\epsilon_{Q}-q\right)$.

Under scope $(T, Q)$ and policy $(t, q)$, the value of social welfare ex post, when the shocks are $\boldsymbol{\epsilon}$ and $\eta$, is then given by:

$$
\begin{aligned}
W^{T, Q}(\boldsymbol{\epsilon}, \eta, t, q)= & \lambda+\sum_{i \in N} \xi_{i}-\frac{t^{2}}{2 b_{T}}-\frac{b_{Q}}{2}\left(z_{Q}+\epsilon_{Q}-q\right)^{2} \\
& -\nu\left(y+\eta+z_{T}+\epsilon_{T}-\frac{t}{b_{T}}-q\right)-\frac{A}{2}\left(y+\eta+z_{T}+\epsilon_{T}-\frac{t}{b_{T}}-q\right)^{2} .
\end{aligned}
$$

The necessary and sufficient FOC for the maximization of the area's social welfare are given by:

$$
\begin{aligned}
& 0=\mathbb{E}\left[\frac{\partial W^{T, Q}}{\partial t}(\boldsymbol{\epsilon}, \eta, t, q)\right]=-\frac{t}{b_{T}}+\frac{\nu}{b_{T}}+\frac{A}{b_{T}}\left(y+z_{T}+q-\frac{t}{b_{T}}\right) \\
& 0=\mathbb{E}\left[\frac{\partial W^{T, Q}}{\partial q}(\boldsymbol{\epsilon}, \eta, t, q)\right]=b_{Q}\left(z_{Q}-q\right)-\nu-A\left(y+z_{T}+q-\frac{t}{b_{T}}\right) .
\end{aligned}
$$

From these, it follows that:

$$
\nu+A\left(y+z_{T}+q-\frac{t}{b_{T}}\right)=t=b_{Q}\left(z_{Q}-q\right)
$$

So, $t=m+\frac{A b_{N} y}{A+b_{N}}$ and $q=x_{Q}^{*}-\frac{A b_{N} y}{b_{Q}\left(A+b_{N}\right)}$. It follows that $x_{N}=q+z_{T}-\frac{t}{b_{T}}+\epsilon_{T}=$ $x_{N}^{*}-\frac{A y}{A+b_{N}}+\epsilon_{T}$ and $p=m+\frac{A b_{N} y}{A+b_{N}}+b_{Q} \epsilon_{Q}$. The expressions collapse to the ones in the text for $y=0$.

\section{Proof of Proposition 2}

The best uniform system has been characterized in the text and it follows that $W^{f b}-$ $W^{\text {unif }}=\frac{A+b_{N}}{2} \mathbb{V}\left[\frac{\mathcal{A}}{A+b_{N}} \epsilon_{N}\right]$. Let us now prove expression (16), calculating the difference between the expression of $W^{f b}-W^{T, Q}$, given by (15), and the previously obtained expression for $W^{f b}-W^{u n i f}$ :

$$
W^{\text {unif }}-W^{T, Q}=\frac{1}{2}\left(A+b_{N}\right)\left\{\mathbb{V}\left[\frac{b_{N}}{A+b_{N}} \epsilon_{N}-\epsilon_{T}\right]-\mathbb{V}\left[\frac{\mathcal{A}}{A+b_{N}} \epsilon_{N}\right]\right\}+\frac{b_{Q} b_{N}}{2 b_{T}} \mathbb{V}\left[\epsilon_{Q}\right]
$$

To compute the difference in variances, we decompose $\epsilon_{N}=\epsilon_{T}+\epsilon_{Q}$ and we develop the terms:

$$
\begin{aligned}
\mathbb{V}\left[\frac{b_{N}}{A+b_{N}} \epsilon_{N}-\epsilon_{T}\right] & -\mathbb{V}\left[\frac{\mathcal{A}}{A+b_{N}} \epsilon_{N}\right]=\frac{1}{\left(A+b_{N}\right)^{2}}\left[\mathbb{V}\left[b_{N} \epsilon_{Q}-A \epsilon_{T}\right]-\mathbb{V}\left[\mathcal{A} \epsilon_{Q}+\mathcal{A} \epsilon_{T}\right]\right] \\
& =\frac{1}{\left(A+b_{N}\right)^{2}}\left\{\left(A^{2}-\mathcal{A}^{2}\right) \mathbb{V}\left[\epsilon_{T}\right]-2\left(A b_{N}+\mathcal{A}^{2}\right) \operatorname{cov}\left(\epsilon_{T}, \epsilon_{Q}\right)+\left(b_{N}^{2}-\mathcal{A}^{2}\right) \mathbb{V}\left[\epsilon_{Q}\right]\right\}
\end{aligned}
$$


Observe that $A^{2}-\mathcal{A}^{2}$ is null for $A=\mathcal{A}$ and equal to $A^{2}-b_{N}^{2}=\left(A-b_{N}\right)\left(A+b_{N}\right)$ for $\mathcal{A}=b_{N}$, hence $A^{2}-\mathcal{A}^{2}=(A-\mathcal{A})\left(A+b_{N}\right)$. Similarly $A b_{N}+\mathcal{A}^{2}$ is either equal to $A\left(b_{N}+A\right)$ for $\mathcal{A}=b_{N}$ and to $\left(A+b_{N}\right) b_{N}$ for $\mathcal{A}=A$, which implies $A b_{N}+\mathcal{A}^{2}=\left(A+b_{N}\right) \mathcal{A}$ and $b_{N}^{2}-\mathcal{A}^{2}=\left(b_{N}-\mathcal{A}\right)\left(A+b_{N}\right)$. Using these in the difference of variances and plugging it into (30) yields (16).

The rest of the proposition, namely the derivation of (17) and (18) is immediate.

\section{Proof of Corollary 3.}

The expected loss of welfare relative to the first best when there are $|T|$ firms in the ETS can easily be computed:

$$
\Omega(|T|)=\frac{\sigma^{2}}{2}\left[\frac{b^{2}}{n A+b}(1-\rho+\rho n)+A|T|(1-\rho+\rho|T|)-b \rho|T|\right] .
$$

This expression is convex quadratic in $|T|$. A uniform ETS is optimal if $\Omega(0) \leq \Omega(1)$, i.e. if $A-b \rho \geq 0$. A uniform tax system is optimal if $\Omega(n) \leq \Omega(n-1)$, i.e. if $\frac{A}{b}(1-2 \rho+2 \rho n) \leq \rho$. Otherwise, the system is mixed and the optimality conditions are given by:

$$
\begin{aligned}
& \Omega(|T|) \leq \Omega(|T|+1) \\
& \Omega(|T|) \leq \Omega(|T|-1) .
\end{aligned}
$$

Simple algebra yields:

$$
A(1-2 \rho+2 \rho|T|) \geq b \rho \geq A(1+2 \rho|T|)
$$

hence the characterization of $|T|$ in the corollary.

\section{Proof of Proposition 3.}

A firm strictly prefers the ETS to the tax if and only if $\operatorname{cov}\left(b_{Q} \widetilde{\epsilon}_{Q}, b_{Q} \widetilde{\epsilon}_{Q}-2 b_{i} \widetilde{\epsilon}_{i}\right)>0$. Assume by contradiction that it is satisfied for each $i$ in $Q$. Dividing by $b_{i}$ and summing over $i$ in $Q$ yields:

$$
\operatorname{cov}\left(b_{Q} \widetilde{\epsilon}_{Q}, \sum_{i \in Q} \frac{b_{Q}}{b_{i}} \widetilde{\epsilon}_{Q}-2 \sum_{i \in Q} \widetilde{\epsilon}_{i}\right)>0 \Leftrightarrow \operatorname{cov}\left(b_{Q} \widetilde{\epsilon}_{Q},-\widetilde{\epsilon}_{Q}\right)>0,
$$

which is impossible.

Proof of (a). Under a common shock $b_{i} \epsilon_{i}=b_{Q} \epsilon_{Q}=\theta$ for all $i$ and the result follows.

Proof of (b). Let shocks have identical variance $\sigma^{2}$, a correlation coefficient $\rho$ and $b_{i}=b$ for all $i$. Then $b_{Q}=\frac{b}{n_{Q}}$ where $n_{Q}$ denotes the number of firms included in the ETS. 
Let $i$ in $Q$. Up to the factor $\sigma^{2}$,

$$
\operatorname{var}\left(\widetilde{\epsilon}_{Q}\right)=n_{Q}\left[1+\left(n_{Q}-1\right) \rho\right] \text { and } \operatorname{cov}\left(b_{Q} \widetilde{\epsilon}_{Q}, b_{i} \widetilde{\epsilon}_{i}\right)=\frac{b^{2}}{n_{Q}}\left[1+\left(n_{Q}-1\right) \rho\right]
$$

This gives:

$$
\operatorname{cov}\left(b_{Q} \widetilde{\epsilon}_{Q}, b_{Q} \widetilde{\epsilon}_{Q}-2 b_{i} \widetilde{\epsilon}_{i}\right)=-\frac{b^{2}}{n_{Q}}\left[1+\left(n_{Q}-1\right) \rho\right]
$$

As $\operatorname{var}\left(\widetilde{\epsilon}_{Q}\right)=n_{Q}\left[1+\left(n_{Q}-1\right) \rho\right]$ can only be non-negative, this proves the result.

\section{Example with a common shock on marginal abatement costs.}

Let us consider the polar situation in which the same shock affects all firms' marginal abatement cost curves, that is: $b_{i} \widetilde{\epsilon}_{i}=\widetilde{\theta}$ for all $i$. The following corollary characterizes the values for which a mixed system is optimal.

Corollary 4. Assume a common shock on marginal abatement costs, $b_{i} \widetilde{\epsilon}_{i}=\widetilde{\theta}$ for all $i$. An optimal scope is a mixed system for $b_{N}<2 A<2 \max _{i \in N} b_{i}$. Up to indivisibilities, an optimal scope satisfies $28=b_{T}$ when $2 A<\max _{i \in N} b_{i}$ and is reduced to a single firm (with maximum $b_{i}$ ) in the taxed sector for $A<\max _{i \in N} b_{i}<2 A$. Otherwise, the optimal scope is a uniform tax system for $b_{N}>2 A$ and a uniform cap-and-trade scheme for $\max _{i \in N} b_{i}<A$.

Proof. When $b_{i} \widetilde{\epsilon}_{i}=\widetilde{\theta}$ for all $i, b_{S} \widetilde{\epsilon}_{S}=\widetilde{\theta}$ for any subset $S \subset N$. Thus, with $\sigma^{2}=\mathbb{V}[\epsilon]$, (16) can be written as:

$$
\begin{aligned}
L(T, Q) & =\sigma^{2}\left\{\frac{A}{b_{T}^{2}}+\frac{1}{b_{Q}}-\mathcal{A}\left[\frac{1}{b_{N}^{2}}\right]\right\} \\
& =\sigma^{2}\left\{\frac{1}{b_{T}}\left(\frac{A}{b_{T}}-1\right)+\frac{1}{b_{N}}\left(1-\frac{\mathcal{A}}{b_{N}}\right)\right\}
\end{aligned}
$$

From this expression, an optimal mixed system must minimize $\frac{1}{b_{T}}\left(\frac{A}{b_{T}}-1\right)$, which, up to indivisibilities, yields $b_{T}=2 A$ and the value $\sigma^{2}\left\{\frac{1}{4 A}+\frac{1}{b_{N}}\left(1-\frac{\mathcal{A}}{b_{N}}\right)\right\}$. This expression must be negative for the mixed system to dominate the uniform systems. The proposition follows.

In the critical case, where $b_{N}=A$, a mixed system is surely optimal (as $b_{N} \leq b_{i}$ for each $i$ ). It is reasonable to assume that there are firms with very small flexibility parameters, i.e. large $b_{i}$, so that $\max _{i \in N} b_{i}$ is very large. Assuming this is the case, a mixed system is optimal whenever $b_{N}<2 A$, and otherwise it is a uniform tax system. In this polar case of a common shock on marginal abatement costs, only the aggregate flexibility of each sector matters, and there is no general result about the values of $b_{i}$ that

\footnotetext{
${ }^{28}$ Any $T$ such that $2 A=b_{T}$ is optimal and, if there is no such $T, b_{T}$ at the optimal scope is either the greatest value smaller than $2 A$ or the smallest one greater than $2 A$.
} 
should be included in the set of firms under the tax or in the ETS, provided the optimal aggregate flexibility in each sector is reached.

\section{Appendix B: A world with several areas}

\section{Worldwide optimum.}

Using the decomposition of $A$ and $\nu$, it follows immediately:

$$
\left\{1+\left(\sum_{\alpha \in \mathcal{W}} A^{\alpha}\right)\left(\sum_{\alpha \in \mathcal{W}} \frac{1}{b_{N^{\alpha}}}\right)\right\} x_{N}^{*}=z_{N}-\left(\sum_{\alpha \in \mathcal{W}} \nu^{\alpha}\right)\left(\sum_{\alpha \in \mathcal{W}} \frac{1}{b_{N^{\alpha}}}\right) .
$$

\section{Proof of Proposition 4.}

1. We compare the situation under merged ETS with the situation under separate ETS, using the notations introduced in the text.

First, note that plugging the value of $x_{i}^{\alpha}$ and $x_{i}$ into the expressions for the profits, we obtain: $\Pi_{i}^{\alpha}(\boldsymbol{\epsilon})=\xi_{i}-\frac{\left(p^{\alpha}\right)^{2}}{2 b_{i}}-p^{\alpha} x_{i}^{\alpha}$ and $\Pi_{i}(\boldsymbol{\epsilon})=\xi_{i}-\frac{(p)^{2}}{2 b_{i}}-p x_{i}$. Summing up over $i \in Q^{\alpha}$, adding up the revenues from the sale of permits and noticing that $\sum_{i \in Q^{\alpha}} x_{i}^{\alpha}=q^{\alpha}$, it comes:

$$
\begin{aligned}
\sum_{i \in Q^{\alpha}} \Pi_{i}^{\alpha}(\boldsymbol{\epsilon})+p^{\alpha} q^{\alpha} & =\sum_{i \in Q^{\alpha}} \xi_{i}-\frac{\left(p^{\alpha}\right)^{2}}{2 b_{Q^{\alpha}}} \\
\sum_{i \in Q^{\alpha}} \Pi_{i}(\boldsymbol{\epsilon})+p q^{\alpha} & =\sum_{i \in Q^{\alpha}} \xi_{i}-\frac{p^{2}}{2 b_{Q^{\alpha}}}+p \sum_{i \in Q^{\alpha}}\left(x_{i}^{\alpha}-x_{i}\right) .
\end{aligned}
$$

Using the fact that $x_{i}^{\alpha}-x_{i}=\frac{p-p^{\alpha}}{b_{i}}$, the difference in social welfare for area $\alpha$ between the situation with merged ETS and that with separate ETS can be written:

$$
\frac{\left(p^{\alpha}\right)^{2}-p^{2}}{2 b_{Q^{\alpha}}}+p \frac{\left(p-p^{\alpha}\right)}{b_{Q^{\alpha}}}=\frac{\left(p^{\alpha}-p\right)^{2}}{2 b_{Q^{\alpha}}} .
$$

This proves Point 1.

2. For given scopes in each area, the general analysis applies at the level of each area $\alpha$ so as to find out this area's best response policy, taking the other areas' random level of emissions $y=x_{N-N^{\alpha}}$ as given.

Let $\mu^{\alpha}(y) \equiv \frac{b_{N^{\alpha}}\left(\nu^{\alpha}+A^{\alpha} y+A^{\alpha} z_{N^{\alpha}}\right)}{A^{\alpha}+b_{N^{\alpha}}}$. Then, the equilibrium tax rate and the optimal aggregate quota in area $\alpha$ given the aggregate emissions volume $x_{N-N^{\alpha}}$ in the other areas are determined by:

$$
\begin{aligned}
t^{\alpha} & =\mu^{\alpha}\left(\mathbb{E}\left[x_{N-N^{\alpha}}\right]\right) \\
q^{\alpha} & =z_{Q^{\alpha}}-\frac{\mu^{\alpha}\left(\mathbb{E}\left[x_{N-N^{\alpha}}\right]\right)}{b_{Q^{\alpha}}}
\end{aligned}
$$


From these, it follows that $p^{\alpha}=\mu^{\alpha}\left(\mathbb{E}\left[x_{N-N^{\alpha}}\right]\right)+b_{Q^{\alpha}} \epsilon_{Q^{\alpha}}$ is the price on area $\alpha$ 's ETS market and $x_{T^{\alpha}}=z_{T^{\alpha}}-\frac{\mu^{\alpha}\left(\mathbb{E}\left[x_{\left.\left.N-N^{\alpha}\right]\right)}\right.\right.}{b_{T^{\alpha}}}+\epsilon_{T^{\alpha}}$. Finally, in terms of the area's aggregate emissions, as a best response:

$$
\mathbb{E}\left[x_{N_{\alpha}}\right]=z_{N^{\alpha}}-\frac{\mu^{\alpha}\left(\mathbb{E}\left[x_{N-N^{\alpha}}\right]\right)}{b_{N^{\alpha}}} .
$$

Since the best response in terms of $\mathbb{E}\left[x_{N_{\alpha}}\right]$ are decreasing with slope of absolute value less than one, a sufficient condition for an interior equilibrium is: for all $\alpha$

$$
z_{N^{\alpha}}-\frac{\mu^{\alpha}(0)}{b_{N^{\alpha}}} \leq \frac{b_{N^{-\alpha}} z_{N^{-\alpha}}-\nu^{-\alpha}}{A^{-\alpha}}
$$

It is then immediate to check that in equilibrium $\mu^{\alpha}\left(\mathbb{E}\left[x_{N-N^{\alpha}}\right]\right)=\nu^{\alpha}+A^{\alpha} \mathbb{E}\left[x_{N}\right]=$ $m^{\alpha}\left(\mathbb{E}\left[x_{N}\right]\right)=b_{i}\left(z_{i}-\mathbb{E}\left[x_{i}\right]\right)$ for any $i \in N^{\alpha}$, so that $\mathbb{E}\left[x_{i}\right]=x_{i}^{e}$ : expected emissions volumes are equal to their value in the equilibrium absent uncertainty. Moreover, the usual manipulation yields:

$$
\left\{1+\left(\sum_{\alpha \in \mathcal{W}} \frac{A^{\alpha}}{b_{N^{\alpha}}}\right)\right\} \mathbb{E}\left[x_{N}\right]=z_{N}-\left(\sum_{\alpha \in \mathcal{W}} \frac{\nu^{\alpha}}{b_{N^{\alpha}}}\right) .
$$

The comparison with the similar expression for $x_{N}^{*}$ is then immediate. Moreover, in equilibrium,

$$
\begin{aligned}
t^{\alpha}=\nu^{\alpha}+A^{\alpha} \mathbb{E}\left[x_{N}\right] & =\nu^{\alpha}+A^{\alpha} x_{N}^{*}+A^{\alpha}\left(\mathbb{E}[x]-x_{N}^{*}\right) \equiv m^{\alpha}\left(x_{N}^{*}\right)+A^{\alpha}\left(\mathbb{E}\left[x_{N}\right]-x_{N}^{*}\right)>m^{\alpha}\left(x_{N}^{*}\right) \\
q^{\alpha} & =z_{Q^{\alpha}}-\frac{t^{\alpha}}{b_{Q^{\alpha}}}=z_{Q^{\alpha}}-\frac{m}{b_{Q^{\alpha}}}+\frac{m-t^{\alpha}}{b_{Q^{\alpha}}}=x_{Q^{\alpha}}^{*}+\frac{m-t^{\alpha}}{b_{Q^{\alpha}}}
\end{aligned}
$$

so that: $q^{\alpha}>x_{Q^{\alpha}}^{*} \Leftrightarrow m>t^{\alpha}$.

Finally, starting from the equilibrium of the complete game of policy choices, let us fix the quotas in all areas at their equilbrium values and consider a reduced game in which areas can only choose their respective tax rates. In this reduced game with separate ETS, it is immediate that the best response tax rate in area $\alpha$ is still given by: $t^{\alpha}=\mu^{\alpha}\left(\mathbb{E}\left[x_{N-N^{\alpha}}\right]\right)$. As this expression only depends upon the tax rates in other areas (through $x_{T^{\beta}}=z_{T^{\beta}}-\frac{t^{\beta}}{b_{T^{\beta}}}+\epsilon_{T^{\beta}}$ for all $\beta \neq \alpha$ ) and the sum of the quotas in the other areas, the same expression also determines the best response tax rate in area $\alpha$ under merged ETS with the sum of quotas. Hence, the same tax rates constitute an equilibrium in the reduced game, whether ETS are separate or merged.

\section{Proof of Proposition 5}

$\alpha$ chooses a uniform cap-and-trade system (resp. a tax system) if $\left(\frac{A}{n}-b\right)+2 \frac{A}{n} \rho n_{t}>0$ where $n_{t}$ is the number of countries choosing a uniform tax system other than $\alpha$. Thus, at equilibrium all countries choose a uniform cap-and-trade if $\frac{A}{n}>b$ (recall $\rho$ is assumed non- 
negative). They all choose a uniform tax if $\left(\frac{A}{n}-b\right)+2 \frac{A}{n} \rho(n-1)<0$. When neither case occurs, we have an equilibrium in which some countries choose the tax, which requires $\left(\frac{A}{n}-b\right)+2 \frac{A}{n} \rho(|\mathcal{T}|-1) \geq 0$ and other choose a cap-and-trade system, which requires $\left(\frac{A}{n}-b\right)+2 \frac{A}{n} \rho|\mathcal{T}| \geq 0$ where $\mathcal{T}$ is the set choosing the tax. These two conditions yield the characterization of $\mathcal{T}$ in the proposition. 